\title{
Global large solutions to the planar magnetohydrodynamics equations with constant heat conductivity
}

Wei $\mathrm{Li}^{1}$ and Zhaoyang Shang ${ }^{1,2^{*}}$ (D)

\author{
"Correspondence: \\ shangzhaoyang@sjtu.edu.cn \\ 'School of Finance, Shanghai Lixin \\ University of Accounting and \\ Finance, Shanghai, P.R. China \\ ${ }^{2}$ School of Mathematical Sciences, \\ Shanghai Jiao Tong University, \\ Shanghai, P.R. China
}

\begin{abstract}
This paper is concerned with global existence of large solutions to the initial-boundary value problem of the planar magnetohydrodynamic compressible flow. Under the assumptions that viscosity and heat conductivity coefficients are constants, magnetic diffusion is a function of the specific volume, we obtain the global existence of strong solutions. Some new methods are developed to deal with the complex interaction between the hydrodynamic and magnetodynamics effects.

Keywords: Planar MHD equations; Global solutions; Constant heat conductivity; Large initial data
\end{abstract}


is the total energy, where $\mathcal{E}^{\prime}=\rho\left(e+\frac{1}{2}|\mathbf{u}|^{2}\right)$ is the energy density of fluid, which is composed of the kinetic energy density $\frac{1}{2} \rho|\mathbf{u}|^{2}$ and the internal energy density $\rho e, \frac{1}{2}|\mathbf{B}|^{2}$ is the magnetic energy. For an ideal polytropic gas, the pressure $p$ and internal energy $e$ are given by the following equations of state:

$$
p=R \rho \theta, \quad e=c_{\nu} \theta,
$$

where $R>0$ is the gas constant, and $c_{v}>0$ is heat capacity at constant volume.

We consider a three-dimensional MHD flow in the domain $[0,1] \times \mathbb{R}^{2}$ with spatial variables $\mathbf{x}=\left(x_{1}, x_{2}, x_{3}\right)$, which is moving in the $x_{1}$ direction and uniform in the transverse direction $\left(x_{2}, x_{3}\right)$, we denote $x=x_{1}$, then we have

$$
\rho=\rho(t, x), \quad \theta=\theta(t, x), \quad \mathbf{u}=(u, \mathbf{w})(t, x), \quad \mathbf{B}=(b, \mathbf{b})(t, x),
$$

where $\mathbf{w}=\left(u_{2}, u_{3}\right)$ is the transverse velocity and $\mathbf{b}=\left(b_{2}, b_{3}\right)$ is the transverse magnetic field; $u$ and $b$ are the longitudinal velocity and longitudinal magnetic field, respectively. From $(1.1)_{4}$ we know that $b$ is a constant and we take $b=1$ without loss of generality. After direct calculations, Eqs. (1.1) can be reduced to the following planar MHD equations in $\Omega=[0,1]$ :

$$
\left\{\begin{array}{l}
\rho_{t}+(\rho u)_{x}=0, \quad x \in \Omega, t>0, \\
(\rho u)_{t}+\left(\rho u^{2}+p+\frac{1}{2}|\mathbf{b}|^{2}\right)_{x}=\left(\lambda u_{x}\right)_{x}, \\
(\rho \mathbf{w})_{t}+(\rho u \mathbf{w}-\mathbf{b})_{x}=\left(\mu \mathbf{w}_{x}\right)_{x}, \\
\mathbf{b}_{t}+(u \mathbf{b}-\mathbf{w})_{x}=\left(\nu \mathbf{b}_{x}\right)_{x}, \\
\mathcal{E}_{t}+\left(u\left(\mathcal{E}^{\prime}+p+|\mathbf{b}|^{2}\right)-\mathbf{w} \cdot \mathbf{b}\right)_{x}=\left(\lambda u u_{x}+\mu \mathbf{w} \cdot \mathbf{w}_{x}+\nu \mathbf{b} \cdot \mathbf{b}_{x}+\kappa \theta_{x}\right)_{x},
\end{array}\right.
$$

where $\lambda=\lambda^{\prime}+2 \mu>0$.

Now, we introduce the Lagrangian variables $(t, y)$, where $y=y(t, x)=\int_{0}^{x} \rho(t, \xi) d \xi$. Without loss of generality, we take $\int_{0}^{1} \rho_{0}(x) d x=1$. Since $y$ is increasing in $x$ and

$$
\int_{0}^{1} \rho(t, x) d x=\int_{0}^{1} \rho_{0}(x) d x=1
$$

we have $y \in[0,1]$. Then Eqs. (1.4) in Euler coordinates $(t, x)$ can be transformed into the following system in Lagrangian coordinates (see [6]):

$$
\left\{\begin{array}{l}
v_{t}-u_{y}=0, \\
u_{t}+\left(p+\frac{1}{2}|\mathbf{b}|^{2}\right)_{y}=\left(\frac{\lambda u_{y}}{v}\right)_{y}, \\
\mathbf{w}_{t}-\mathbf{b}_{y}=\left(\frac{\mu \mathbf{w}_{y}}{v}\right)_{y}, \\
(\nu \mathbf{b})_{t}-\mathbf{w}_{y}=\left(\frac{v \mathbf{b}_{y}}{v}\right)_{y}, \\
\mathcal{E}_{t}+\left(u\left(p+\frac{1}{2}|\mathbf{b}|^{2}\right)-\mathbf{w} \cdot \mathbf{b}\right)_{y}=\left(\frac{\lambda u u_{y}}{v}+\frac{\mu \mathbf{w} \cdot \mathbf{w}_{y}}{v}+\frac{\nu \mathbf{b} \cdot \mathbf{b}_{y}}{v}+\frac{\kappa \theta_{y}}{v}\right)_{y},
\end{array}\right.
$$

where the total energy of the planar magnetohydrodynamic flow is

$$
\mathcal{E}=e+\frac{1}{2}\left(u^{2}+|\mathbf{w}|^{2}+v|\mathbf{b}|^{2}\right),
$$


and the equations of state (1.2) become

$$
p=R \frac{\theta}{v}, \quad e=c_{\nu} \theta
$$

Moreover, from (1.6), we have the temperature equation

$$
\left(c_{v} \theta\right)_{t}+p u_{y}=\frac{\lambda}{v} u_{y}^{2}+\frac{\mu}{v}\left|\mathbf{w}_{y}\right|^{2}+\frac{v}{v}\left|\mathbf{b}_{y}\right|^{2}+\left(\frac{\kappa \theta_{y}}{v}\right)_{y}
$$

In this paper, we consider the initial-boundary value problem of (1.6) in a bounded spatial domain $\Omega=[0,1]$ with the following initial and impermeable, thermally isolated boundary conditions:

$$
\begin{aligned}
& \left.(v, u, \mathbf{w}, \mathbf{b}, \theta)\right|_{t=0}=\left(v_{0}, u_{0}, \mathbf{w}_{0}, \mathbf{b}_{0}, \theta_{0}\right)(y), \quad y \in \Omega, \\
& \left.\left(u, \mathbf{w}, \mathbf{b}, \theta_{y}\right)\right|_{\partial \Omega}=0
\end{aligned}
$$

where the initial data satisfy compatibility conditions.

There have been a lot of studies on MHD equations according to the efforts of physicists and mathematicians because of its physical application and mathematical being challenging, see $[4,5,12,15,17,23,24,35]$ and the references cited therein. First, let us recall some well-posedness results of (1.6). In 1972, Vol'pert and Hudjaev first proved the existence and uniqueness of local smooth solutions in their famous paper [39]. In 1982, Kawashima and Okada [23] showed the existence of global smooth solutions with small initial data. For large initial data, the pioneering work can be traced back to Kazhikhov [26] for the following simplest one-dimensional MHD equations in 1987:

$$
\left\{\begin{array}{l}
v_{t}-u_{y}=0, \\
u_{t}+\left(p+\frac{1}{2}|b|^{2}\right)_{y}=\left(\frac{\lambda u_{y}}{v}\right)_{y}, \\
(v b)_{t}=\left(\frac{v b_{y}}{v}\right)_{y} \\
\theta_{t}+p u_{y}=\frac{\lambda}{v} u_{y}^{2}+\frac{v}{v}\left|b_{y}\right|^{2}+\left(\frac{\kappa \theta_{y}}{v}\right)_{y},
\end{array}\right.
$$

with the initial and boundary conditions:

$$
\begin{aligned}
& \left.(v, u, b, \theta)\right|_{t=0}=\left(v_{0}, u_{0}, b_{0}, \theta_{0}\right)(y), \quad y \in(0,1), \\
& \left.\left(u, b_{y}, \theta_{y}\right)\right|_{y=0,1}=0 .
\end{aligned}
$$

He proved the global existence of strong solutions when all coefficients are positive constants. Under the growth condition

$$
\underline{\kappa}\left(1+\theta^{q}\right) \leq \kappa(\theta) \leq \bar{\kappa}\left(1+\theta^{q}\right)
$$

Zhang and Zhao [43] obtained the global strong solution of (1.11) with $q \geq 0, v=0$, and the non-resistive limit result with $q>0, v>0$ when the initial density is strictly positive. Recently, Li and Jiang [30] obtained the global well-posedness of strong and weak solutions to the Cauchy problem of (1.11) with $v=0$. 
Remark 1.1 When we take $\mathbf{u}=(0,0, u)(t, x), \mathbf{B}=(0,0, b)(t, x)$ in (1.1), we have the following simplest one-dimensional MHD equations in Euler coordinates:

$$
\left\{\begin{array}{l}
\rho_{t}+(\rho u)_{x}=0, \\
(\rho u)_{t}+\left(\rho u^{2}+p+\frac{1}{2} b^{2}\right)_{x}=\lambda u_{x x}, \\
b_{t}+(u b)_{x}=v b_{x x}, \\
(\rho \theta)_{t}+(\rho u \theta)_{x}+p u_{x}=\lambda u_{x}^{2}+\nu b_{y}^{2}+\left(\kappa \theta_{x}\right)_{x},
\end{array}\right.
$$

from which one deduces (1.11) after a Lagrangian transformation as mentioned in planar MHD equations.

Compared with (1.11), due to the coupling between transverse velocity and transverse magnetic field in (1.6), we cannot get $\mathbf{b} \in L^{\infty}\left([0, T] ; L^{\infty}(\Omega)\right)$ directly, which is one of the difficulties in planar MHD equations. Now, let us turn back to recall some known results of (1.6) with variable and constant magnetic diffusion, which the heat conductivity depend on temperature. In 2002, under some growth conditions of pressure and internal energy, the existence and uniqueness of global strong solutions were proved by Chen and Wang for free boundary problem [6] and by Wang for the initial-boundary value problem [40], when the heat conductivity $\kappa(\nu, \theta)$ satisfies

$$
\kappa_{0}\left(1+\theta^{q}\right) \leq \kappa(v, \theta) \leq \kappa_{1}\left(1+\theta^{q}\right), \quad\left|\kappa_{v}(v, \theta)\right|+\left|\kappa_{v v}(v, \theta)\right| \leq \kappa_{1}\left(1+\theta^{q}\right),
$$

for $q \geq 2$, and the viscosities $\lambda, \mu$, magnetic diffusion $v$ satisfy

$$
\lambda_{0} \leq \lambda(v) \leq \lambda_{1}, \quad \mu_{0} \leq \mu(v) \leq \mu_{1}, \quad v_{0} \leq v(v) \leq v_{1},
$$

where $\kappa_{i}, \lambda_{i}, \mu_{i}, v_{i}(i=0,1)$ are some positive constants. Chen and Wang [7] also proved the Lipschitz continuous dependence of solutions on the initial data for perfect gas when coefficients are independent of the specific volume. For global weak solutions, we refer to $[15,16,19]$ and the references cited therein. Recently, under the assumption

$$
\kappa(\theta)=\tilde{\kappa} \theta^{q},
$$

for $q>0, \mathrm{Hu}$ and $\mathrm{Ju}[20]$ proved the global existence of strong solutions to initial-boundary value problem (1.6)-(1.10), which was extended by Fan et al. [14] to the case when the initial data allow for vacuum. Also see [13] when transverse velocity is not of concern and the resistivity coefficient is taken $v=0$. Estimates on the thickness of the boundary layer and vanishing shear viscosity limit were studied by Qin et al. in [35]. For planar MHD equations coupled to self-gravitation and radiation effects, the global existence of a classical solution can be found in [42]. Very recently, Li studied non-isentropic and isentropic planar MHD equations in Refs. [28] and [29], respectively, where global strong solutions with the initial density is bounded below away from vacuum and allowed vacuum are proved when the resistivity coefficient $v=0$.

Now, we make some remarks on the proof of global existence when the heat conductivity is a power function of the temperature. The key point is to obtain the upper bound of the specific volume. Conditions (1.16) with $q \geq 2$ in $[6,7,40]$ and condition (1.18) with $q>0$ 
in [20] are crucial in the entropy estimate to obtain the upper bounds of the $\|\theta\|_{L^{1-\alpha}\left(0, T ; L^{\infty}\right)}$ and $\|\mathbf{b}\|_{L^{2}\left(0, T ; L^{\infty}\right)}$, for any $0 \leq \alpha \leq 1$. In fact, these two norms can be used to give the representation of the specific volume by the classical method proposed in [27]. In other words, the upper bound of the specific volume can be achieved through the upper bounds of the above two norms. Moreover, after having the upper bound of $v,\|\theta\|_{L^{1-\alpha}\left(0, T ; L^{\infty}\right)}$ can be improved to $\|\theta\|_{L^{1+\alpha}\left(0, T ; L^{\infty}\right)}$, from which, combined with the momentum equation, estimates on the first-order derivative of specific volume can be established. Then there follow the estimates on the higher-order derivatives of solutions by using the standard energy method.

However, the results about the global existence of planar MHD equations with constant heat conductivity (the case $q=0$ ) are very limited, even though the corresponding problem for the Navier-Stokes equations was solved in [27] a long time ago. For local and global existence of strong solutions to the Navier-Stokes equations with constant viscosity and heat conductivity, we refer to $[9,10,21,22,25,27,31-33,38]$ and the references cited therein. Our mission in this paper is to establish the global existence of strong solutions to (1.6)-(1.10) when $\mu, \lambda, \kappa$ are constants and the magnetic diffusion $v(v)$ is a function of $v$ with first-order derivative continuity satisfying

$$
\lim _{v \rightarrow+\infty} \frac{v}{v(v)}<+\infty
$$

Remark 1.2 When the conducting fluid has free electrons inside, the induced current is formed under the action of the electromagnetic field, which produces Joule heat and makes the electromagnetic energy consume. Then the electromagnetic energy is converted into heat energy during the transmission process. When the fluid is close to vacuum, the magnetic flux propagates with almost no attenuation and speeds up the magnetic-diffusion process. In this sense, condition (1.19) is reasonable and it will be used to yield some estimates on the upper bound for the specific volume.

We now make some comments on the global existence of large solutions to planar MHD equations with heat conductivity in the form of (1.18) for $q=0$. Compared to the case of the temperature-dependent heat conductivity, it causes some essential difficulties. From the point of view of physics, the dissipative effect decreases for the high temperature. Mathematically, the power relation between the heat conductivity and the temperature is lost, which is essentially needed to derive the upper bounds of the specific volume and the temperature. The main difficulties in the proof is to obtain the upper bound of the specific volume and the estimate on the first-order derivative of the specific volume. More precisely, our analysis can be outlined as follows:

(1) Upper bound of the specific volume.

When the heat conductivity is a constant, the regularity of both the temperature and the magnetic field cannot be improved in the same way as in the temperature-dependent case. Hence, the methods proposed in Ref. [6, 20, 40] are no longer applicable. Therefore, new techniques need to be developed. We first give a representation of the specific volume in terms of the full pressure $\left(p+\frac{|\mathbf{b}|^{2}}{2}\right)$ to get the lower bounds of $v$ and $\theta$. Second, we obtain an entropy-type energy estimate involving the dissipative effects of viscosity, magnetic diffusion, and heat diffusion, from which, $\|\theta\|_{L^{1-\alpha}\left(0, T ; L^{\infty}\right)}$ can be obtained when $\alpha$ is close to 1 . Then the 
combination of the entropy estimate and the assumption (1.19) implies $\|\mathbf{b}\|_{L^{2}\left(0, T ; L^{\infty}\right)}$. With these regularities of the temperature and the magnetic field, the upper bound of the specific volume can be obtained.

(2) Estimate on the first-order derivative of the specific volume.

On the other hand, due to the restriction of the narrow range of $\alpha$ in the entropy estimate, $\|\theta\|_{L^{1+\alpha}\left(0, T ; L^{\infty}\right)}$ cannot be estimated for any $0 \leq \alpha \leq 1$ as well as in temperature-dependent case. Hence, we cannot obtain the bound of $\left\|v_{y}(t)\right\|_{L^{2}}$ directly, which is essential in deriving the upper bound of the temperature and the higher-order estimates of solutions. To overcome such difficulties, we give a new representation of $\left\|v_{y}(t)\right\|_{L^{2}}$ from the momentum equation (1.6) 2 , in which we find that only $\left\|\theta_{y}\right\|_{L^{2}\left(0, T ; L^{2}\right)}$ need to be estimated. Then we turn to the temperature equations (1.8), however, due to the complex interaction between the hydrodynamic and magnetodynamic effects, we need additional estimates on $\left\|\theta u_{y}^{2}\right\|_{L^{1}\left(0, T ; L^{1}\right)}$, $\left\|\theta \mathbf{w}_{y}^{2}\right\|_{L^{1}\left(0, T ; L^{1}\right)}$ and $\left\|\theta \mathbf{b}_{y}^{2}\right\|_{L^{1}\left(0, T ; L^{1}\right)}$ with temperature as weight. For this, we propose some new estimates to deal with the cross term of the fluid and the magnetic field.

In the following theorem we show that (1.6)-(1.10) admit a unique global strong solution when the initial data $\left(v_{0}, u_{0}, \mathbf{w}_{0}, \mathbf{b}_{0}, \theta_{0}\right)(y)$ belong to $H^{1} \times H^{2} \times H^{2} \times H^{2} \times H^{2}$. Furthermore, the solution could be classical if the initial data have better regularity.

Theorem 1.1 Suppose that viscosities $\mu, \lambda$, heat conductivity $\kappa$ are constants and magnetic diffusion $v=v(v)$ satisfies (1.19). If the initial data $\left(v_{0}, u_{0}, \mathbf{w}_{0}, \mathbf{b}_{0}, \theta_{0}\right)(y)$ satisfy

$$
v_{0} \in H^{1}, \quad\left(u_{0}, \mathbf{w}_{0}, \mathbf{b}_{0}, \theta_{0}\right) \in H^{2},
$$

and there exists a positive constant $C_{0}$ such that

$$
C_{0}^{-1} \leq v_{0}(y) \leq C_{0}, \quad C_{0}^{-1} \leq \theta_{0}(y) \leq C_{0},
$$

then (1.6)-(1.10) admit a unique global strong solution $(v, u, \mathbf{w}, \mathbf{b}, \theta)(y, t)$ such that, for any fixed $T>0$,

$$
v \in L^{\infty}\left(0, T ; H^{1}\right), \quad(u, \mathbf{w}, \mathbf{b}, \theta) \in L^{\infty}\left(0, T ; H^{2}\right)
$$

and, for any $(y, t) \in[0,1] \times[0, T]$,

$$
\begin{aligned}
& C^{-1} \leq v(y, t) \leq C, \quad C^{-1} \leq \theta(y, t) \leq C, \\
& \|v(\cdot, t)\|_{H^{1}}+\|(u, \mathbf{w}, \mathbf{b}, \theta)(\cdot, t)\|_{H^{2}} \leq C, \\
& \int_{0}^{1}\left(u_{t}^{2}+\left|\mathbf{w}_{t}\right|^{2}+\left|\mathbf{b}_{t}\right|^{2}+\theta_{t}^{2}\right) d y+\int_{0}^{T} \int_{0}^{1}\left(u_{y t}^{2}+\left|\mathbf{w}_{y t}\right|^{2}+\left|\mathbf{b}_{y t}\right|^{2}+\theta_{y t}^{2}\right) d y d t \leq C,
\end{aligned}
$$

where $C>0$ is a constant depending only on the initial data, $T$ and the parameters of the system.

Remark 1.3 If in addition the initial data $\left(v_{0}, u_{0}, \mathbf{w}_{0}, \mathbf{b}_{0}, \theta_{0}\right)(y)$ satisfy

$$
v_{0}(y) \in C^{1+\eta}, \quad\left(u_{0}, \mathbf{w}_{0}, \mathbf{b}_{0}, \theta_{0}\right)(y) \in C^{2+\eta},
$$


for some $\eta \in(0,1)$, then (1.6)-(1.10) admit a unique global classical solution such that, for any fixed $T>0$,

$$
v(y, t) \in C^{1+\eta, \frac{\eta}{2}}, \quad(u, \mathbf{w}, \mathbf{b}, \theta)(y, t) \in C^{2+\eta, 1+\frac{\eta}{2}},
$$

and for any $(y, t) \in[0,1] \times[0, T],(v, u, \mathbf{w}, \mathbf{b}, \theta)(y, t)$ satisfies $(1.21)-(1.23)$.

Remark 1.4 As a special case of (1.19), in this paper we can take

$$
v(v)=C v^{\gamma}, \quad \gamma \geq 1,
$$

which satisfies the following condition (1.27) given by Guo and Xie [18], they studied the existence of global weak solution for a generalized quantum MHD equations and gave a physically grounded assumption that magnetic diffusion is a continuous function of the specific volume and there exists $B>0$ such that

$$
\left\{\begin{array}{l}
d_{0} v^{a} \leq v(v) \leq d_{0}^{\prime} v^{a^{\prime}}, \quad v>B, \\
d_{1} \leq v(v) \leq d_{1}^{\prime} v^{-b}, \quad v \leq B,
\end{array}\right.
$$

where positive constants $d_{0}, d_{0}^{\prime}, d_{1}, d_{1}^{\prime}$ large enough, $2 \leq a<a^{\prime}<3$ and $b \in[0, \infty]$. In this paper we do not need any restrictive conditions as (1.17) and (1.27) which are imposed on the upper bound of the magnetic diffusion. For similar restrictive conditions we refer to $[1,2,37]$, and the references therein.

Remark 1.5 When a more detailed description about magnetohydrodynamics is needed, for instance, whenever one deals with phenomena with characteristic length scales comparable or smaller than the ion skin depth, $d_{i}=c / \omega_{p i}$, with $\omega_{p i}$ as the ion plasma frequency, $c$ is speed of light, the Hall effect which takes into account the relation between electrons and ions cannot be neglected and we need Hall-MHD equations to describe this kind of flow. A well-known reason is that, for scales below $d_{i}$, it becomes inaccurate to make the approximation that the magnetic field is carried by the bulk flow of ions. From the structure of the following planar Hall-MHD equations:

$$
\left\{\begin{array}{l}
v_{t}+u_{y}=0, \\
u_{t}+\left(p+\frac{1}{2}|\mathbf{b}|^{2}\right)_{y}=\left(\frac{\lambda u_{y}}{v}\right)_{y}, \\
\mathbf{w}_{t}-\mathbf{b}_{y}=\left(\frac{\mu \mathbf{w}_{y}}{v}\right)_{y}, \\
(\nu \mathbf{b})_{t}-\mathbf{w}_{y}=\left(\frac{\nu \mathbf{b}_{y}}{v}\right)_{y}+\left(\epsilon A \mathbf{b}_{y}\right)_{y}, \\
E_{t}+\left(u\left(p+\frac{1}{2}|\mathbf{b}|^{2}\right)-\mathbf{w} \cdot \mathbf{b}\right)_{y}=\left(\frac{\lambda u u_{y}}{v}+\frac{\mu \mathbf{w} \cdot \mathbf{w}_{y}}{v}+\frac{\nu \mathbf{b} \cdot \mathbf{b}_{y}}{v}+\epsilon A^{\top} \mathbf{b} \cdot \mathbf{b}_{y}+\frac{\kappa \theta_{y}}{v}\right)_{y},
\end{array}\right.
$$

where $\epsilon$ is a Hall parameter, $A$ is the constant coefficient matrix

$$
A=\left(\begin{array}{cc}
0 & 1 \\
-1 & 0
\end{array}\right)
$$

and $A^{\top}$ is the transpose of the matrix $A$, we can see that similar results to Theorem 1.1 and Remark 1.3 can be obtained by using the techniques of this paper with some modifications. 
At the end of this paper, we will give a brief derivation of model (1.28) and prove the global existence of a strong solution for the planar Hall-MHD equations (1.28) with initialboundary conditions (1.9) and (1.10).

The existence of local solution can be obtained by the standard method based on the Banach fixed point theorem on a small time interval (cf. [31]). The global existence of strong solution will be proved by the method of extending the local solution with respect to time through the a priori global estimates.

The rest of this paper is organized as follows. In Sect. 2, we establish the a priori estimates on the bounds of the specific volume. In Sect. 3, we focus on the $L^{2}$-norm of $v_{y}$, then estimates on the first-order derivatives of solutions are obtained. In Sect. 4, we get the estimates on the higher-order derivatives of solutions by standard energy method. From the a priori estimates proved above and continuation argument, we extend the local solution to the global one. Finally, in Sect. 5, global existence of strong solution for Hall-MHD equations is proved for the completeness of Remark 1.5.

Notation For simplicity of the presentation, we take $\mu=\lambda=\kappa=R=c_{\nu}=1$. Based on the local existence theorem, we assume that $(v, u, \mathbf{w}, \mathbf{b}, \theta)(y, t)$ is the unique strong solution of (1.6)-(1.10) defined on $[0,1] \times[0, T]$ for some $T>0$. In the following, $C>0$ denotes a generic constant, which may depend on $T$ and may vary from place to place.

\section{A priori estimates on the bounds of the specific volume}

Now, we will derive a sequence of a priori estimates in the following lemmas to prove Theorem 1.1. In this section, we first give a representation of $v$ and obtain the lower bound of $v$ and $\theta$ in Lemma 2.1. Then, through careful analysis, we obtain an entropy-type estimate and the upper bound of $v$ in Lemma 2.2 and Lemma 2.3, respectively.

First of all, from (1.6), we obtain the conservation of mass and energy

$$
\begin{aligned}
& \int_{0}^{1} v d y=\int_{0}^{1} v_{0} d y \leq C, \\
& \int_{0}^{1}\left(\theta+\frac{1}{2}\left(u^{2}+|\mathbf{w}|^{2}+v|\mathbf{b}|^{2}\right)\right) d y=\int_{0}^{1}\left(\theta_{0}+\frac{1}{2}\left(u_{0}^{2}+\left|\mathbf{w}_{0}\right|^{2}+v_{0}\left|\mathbf{b}_{0}\right|^{2}\right)\right) d y \leq C .
\end{aligned}
$$

In the following lemma, we use Kazhikhov's method [27] to derive a representation of the specific volume $v$ and prove the lower bounds of $v$ and $\theta$, which lay the foundation of the global existence.

Lemma 2.1 There exists a positive constant $C>0$ such that, for any $(y, t) \in[0,1] \times[0, T]$,

$$
\begin{aligned}
& v(y, t) \geq C^{-1}, \\
& \theta(y, t) \geq C^{-1} .
\end{aligned}
$$

Proof The proof will be divided into three steps.

Step 1. Representation of the specific volume $v$.

We set

$$
h(y, t)=\int_{0}^{t}\left(\frac{u_{y}}{v}-p-\frac{1}{2}|\mathbf{b}|^{2}\right)(y, s) d s+\int_{0}^{y} u_{0}(\xi) d \xi .
$$


Equation $(1.6)_{2}$ implies $h_{y}=u$, then we have

$$
h(y, t)=h\left(a_{1}(t), t\right)+\int_{a_{1}(t)}^{y} u(\xi, t) d \xi
$$

where $a_{1}(t) \in[0,1]$ is a function of $t$ and will be determined later. On the other hand, since $u_{y}=v_{t}$, then

$$
h(y, t)=\ln v-\ln v_{0}-\int_{0}^{t}\left(p+\frac{1}{2}|\mathbf{b}|^{2}\right)(y, s) d s+\int_{0}^{y} u_{0}(\xi) d \xi
$$

we have

$$
v^{-1} \exp \left(\int_{0}^{t} p(y, s) d s\right)=\exp \left(A(y, t)-\int_{0}^{t} \frac{1}{2}|\mathbf{b}|^{2} d s\right)
$$

where

$$
A(y, t)=\int_{0}^{y} u_{0}(\xi) d \xi-h\left(a_{1}(t), t\right)-\int_{a_{1}(t)}^{y} u(\xi, t) d \xi-\ln v_{0}
$$

Multiplying $\theta$ on both sides of Eq. (2.7) and integrating the result over $[0, t]$, we get

$$
\int_{0}^{t}\left(p(y, s) \exp \left(\int_{0}^{s} p(y, \tau) d \tau\right)\right) d s=\int_{0}^{t} \theta(y, s) \exp \left(A(y, s)-\int_{0}^{s} \frac{1}{2}|\mathbf{b}|^{2} d \tau\right) d s,
$$

then

$$
\exp \left(\int_{0}^{t} p(y, s) d s\right)-1=\int_{0}^{t} \theta(y, s) \exp \left(A(y, s)-\int_{0}^{s} \frac{1}{2}|\mathbf{b}|^{2} d \tau\right) d s
$$

Hence, we conclude

$$
v=\exp \left(\int_{0}^{t} \frac{1}{2}|\mathbf{b}|^{2} d s-A(y, t)\right)\left(1+\int_{0}^{t} \theta(y, s) \exp \left(A(y, s)-\int_{0}^{s} \frac{1}{2}|\mathbf{b}|^{2} d \tau\right) d s\right) .
$$

Step 2. Lower bound of the specific volume $v$.

We now determine $a_{1}(t)$. From the definition of $h$, we obtain

$$
h_{t}=\frac{u_{y}}{v}-p-\frac{1}{2}|\mathbf{b}|^{2} .
$$

Then, using $v_{t}=u_{y}$ and $h_{y}=u$, we get

$$
(v h)_{t}=(u+u h)_{y}-\left(u^{2}+v p+\frac{1}{2} v|\mathbf{b}|^{2}\right)
$$

Integrating the above equation over $[0,1] \times[0, t]$ and together with

$$
h(y, 0)=\int_{0}^{y} u_{0}(\xi) d \xi
$$


it yields

$$
\int_{0}^{1} v h(y, t) d y=\int_{0}^{1} v_{0}(y) \int_{0}^{y} u_{0}(\xi) d \xi d y-\int_{0}^{t} \int_{0}^{1}\left(u^{2}+v p+\frac{1}{2} v|\mathbf{b}|^{2}\right)(y, s) d y d s
$$

We assume that

$$
\int_{0}^{1} v(y, t) d y=\int_{0}^{1} v_{0}(y) d y=\bar{v}_{0}
$$

then we set $a_{1}(t) \in[0,1]$ satisfying

$$
h\left(a_{1}(t), t\right)=\frac{1}{\bar{v}_{0}} \int_{0}^{1} v h(y, t) d y .
$$

Hence, we have

$$
\begin{aligned}
v(y, t)= & Y(y, t) \exp \left(\int_{0}^{t} Q(y, s) d s\right) \\
& +\int_{0}^{t} \theta(y, s) Y(y, t) Y(y, s)^{-1} \exp \left(\int_{s}^{t} Q(y, \tau) d \tau\right) d s
\end{aligned}
$$

where

$$
Y(y, t)=\exp \left(\frac{1}{\bar{v}_{0}} \int_{0}^{1} v_{0}(y) \int_{0}^{y} u_{0}(\xi) d \xi d y+\int_{a_{1}(t)}^{y} u(\xi, t) d \xi-\int_{0}^{y} u_{0}(\xi) d \xi+\ln v_{0}\right)
$$

and

$$
Q(y, t)=\frac{1}{2}|\mathbf{b}|^{2}-\int_{0}^{1}\left(u^{2}+v p+\frac{1}{2} v|\mathbf{b}|^{2}\right) d y .
$$

Since

$$
\left|\int_{a_{1}(t)}^{y} u(\xi, t) d \xi\right| \leq\left(\int_{0}^{1} u^{2} d y\right)^{\frac{1}{2}} \leq C,
$$

it is easy to see that

$$
C^{-1} \leq Y(y, t) \leq C .
$$

Equation (2.2) implies that $Q(y, t) \geq C$. Hence, for any $(y, t) \in[0,1] \times[0, T]$ we have

$$
v(y, t) \geq C^{-1} .
$$

Step 3. Lower bound of the temperature $\theta$.

Multiplying (1.8) by $\theta^{-2}$, we have

$$
\left(\frac{1}{\theta}\right)_{t}=\left(\frac{1}{v}\left(\frac{1}{\theta}\right)_{y}\right)_{y}-\left(\frac{2 \theta_{y}^{2}}{v \theta^{3}}+\frac{1}{v \theta^{2}}\left(u_{y}-\frac{\theta}{2}\right)^{2}+\frac{\mathbf{w}_{y}^{2}+v(v) \mathbf{b}_{y}^{2}}{v \theta^{2}}\right)+\frac{1}{4 v} .
$$


Then, multiplying the above equation by $2 r\left(\frac{1}{\theta}\right)^{2 r-1}$, integrating the result in $y$ over $[0,1]$ and using the boundary condition (1.10), we have

$$
\begin{aligned}
& \int_{0}^{1} 2 r\left(\frac{1}{\theta}\right)^{2 r-1}\left(\frac{1}{\theta}\right)_{t} d y \\
& \quad \leq \int_{0}^{1} 2 r\left(\frac{1}{\theta}\right)^{2 r-1}\left(\frac{1}{v}\left(\frac{1}{\theta}\right)_{y}\right)_{y} d y+\int_{0}^{1} \frac{r}{2 v}\left(\frac{1}{\theta}\right)^{2 r-1} d y \\
& \quad \leq-\int_{0}^{1} 2 r(2 r-1)\left(\frac{1}{\theta}\right)^{2 r-2} \frac{1}{v}\left(\frac{1}{\theta}\right)_{y}^{2} d y+\frac{r}{2} \int_{0}^{1} \frac{1}{v}\left(\frac{1}{\theta}\right)^{2 r-1} d y \\
& \leq \frac{r}{2} \int_{0}^{1} \frac{1}{v}\left(\frac{1}{\theta}\right)^{2 r-1} d y
\end{aligned}
$$

where $r$ is a natural number. Using the Hölder inequality, it is easy to see that

$$
\frac{d}{d t}\left\|\frac{1}{\theta}\right\|_{L^{2 r}}^{2 r} \leq \frac{r}{2}\left\|\frac{1}{\theta}\right\|_{L^{2 r}}^{2 r-1}\left\|\frac{1}{v}\right\|_{L^{2 r}}
$$

then we have

$$
\left\|\frac{1}{\theta}\right\|_{L^{2 r}} \leq C
$$

Let $r \rightarrow \infty$, we can get $\theta \geq C^{-1}$. This completes the proof of Lemma 2.1 .

In order to explore the dissipation mechanism of compressible magnetohydrodynamics equations and obtain an entropy-type estimate, we recall Gibb's equation

$$
\theta d S=d e+p d \nu
$$

From equations of the state for perfect gas imposed in (1.7), one can choose the specific entropy

$$
S=\ln \theta+\ln v
$$

With the aid of $(1.6)_{1}$ and (1.8), we have

$$
S_{t}=\frac{\theta_{t}}{\theta}+\frac{v_{t}}{v}=\left(\frac{\theta_{y}}{v \theta}\right)_{y}+\frac{\theta_{y}^{2}}{v \theta^{2}}+\frac{u_{y}^{2}}{\nu \theta}+\frac{\left|\mathbf{w}_{y}\right|^{2}}{\nu \theta}+\frac{\nu(v)\left|\mathbf{b}_{y}\right|^{2}}{\nu \theta}
$$

Next, we give the following entropy estimate, from which, $\|\theta\|_{L^{1-\alpha}\left(0, T ; L^{\infty}\right)}$ can be obtained when $\alpha$ is close to 1 . It is essential in our proof.

Lemma 2.2 There exist positive constants $C>0$ and $0<\beta<1$, such that, for any $\alpha \in(\beta, 1]$, $t \in[0, T]$,

$$
\int_{0}^{t} \int_{0}^{1} \frac{\alpha \theta_{y}^{2}}{v \theta^{\alpha+1}} d y d s+\int_{0}^{t} \int_{0}^{1} \frac{u_{y}^{2}+\left|\mathbf{w}_{y}\right|^{2}+v(v)\left|\mathbf{b}_{y}\right|^{2}}{v \theta^{\alpha}} d y d s+\int_{0}^{t} \max _{y \in[0,1]} \theta^{1-\alpha} d s \leq C
$$


Li and Shang Boundary Value Problems

(2019) 2019:162

Page 12 of 31

Proof We first prove the case $\alpha=1$. Integrating $(2.19)$ over $[0,1] \times[0, t]$, we get

$$
\int_{0}^{t} \int_{0}^{1}\left(\frac{u_{y}^{2}}{v \theta}+\frac{\left|\mathbf{w}_{y}\right|^{2}}{v \theta}+\frac{v(v)\left|\mathbf{b}_{y}\right|^{2}}{v \theta}+\frac{\theta_{y}^{2}}{v \theta^{2}}\right) d y d s=\int_{0}^{1} S(y, t) d y-\int_{0}^{1} S(y, 0) d y .
$$

It follows from (2.1) and (2.2) that

$$
\int_{0}^{1} S(y, t) d y \leq \int_{0}^{1}(\theta+v) d y \leq C,
$$

where we have used the fact $\ln x \leq x, x>0$.

For the case $0<\beta<\alpha<1$, where $\beta$ will be determined later. Multiplying (1.8) by $-\theta^{-\alpha}$ and integrating the resulting equation over $[0,1] \times[0, t]$, we have

$$
\begin{aligned}
& \int_{0}^{t} \int_{0}^{1} \frac{\alpha \theta_{y}^{2}}{v \theta^{\alpha+1}} d y d s+\int_{0}^{t} \int_{0}^{1} \frac{u_{y}^{2}+\left|\mathbf{w}_{y}\right|^{2}+v(v)\left|\mathbf{b}_{y}\right|^{2}}{v \theta^{\alpha}} d y d s \\
& \quad=\frac{1}{1-\alpha}\left(\int_{0}^{1} \theta^{1-\alpha} d y-\int_{0}^{1} \theta_{0}^{1-\alpha} d y\right)+\int_{0}^{t} \int_{0}^{1} p u_{y} \theta^{-\alpha} d y d s \\
& \quad \leq C+\int_{0}^{t} \int_{0}^{1} p u_{y} \theta^{-\alpha} d y d s .
\end{aligned}
$$

By using the Holder inequality, the terms on the right-hand side of the above equation can be estimated as

$$
\begin{aligned}
\int_{0}^{t} \int_{0}^{1} p u_{y} \theta^{-\alpha} d y d s & \leq \int_{0}^{t}\left(\int_{0}^{1} \frac{u_{y}^{2}}{v \theta^{\alpha}} d y\right)^{\frac{1}{2}}\left(\int_{0}^{1} \frac{p^{2} v}{\theta^{\alpha}} d y\right)^{\frac{1}{2}} d s \\
& \leq \frac{1}{2} \int_{0}^{t} \int_{0}^{1} \frac{u_{y}^{2}}{v \theta^{\alpha}} d y d s+\frac{1}{2} \int_{0}^{t} \int_{0}^{1} \frac{1}{v \theta^{\alpha-2}} d y d s \\
& \leq \frac{1}{2} \int_{0}^{t} \int_{0}^{1} \frac{u_{y}^{2}}{v \theta^{\alpha}} d y d s+\frac{1}{2} \int_{0}^{t} \max _{y \in[0,1]} \theta^{1-\alpha} \int_{0}^{1} \frac{\theta}{v} d y d s \\
& \leq \frac{1}{2} \int_{0}^{t} \int_{0}^{1} \frac{u_{y}^{2}}{v \theta^{\alpha}} d y d s+C \int_{0}^{t} \max _{y \in[0,1]} \theta^{1-\alpha} d s .
\end{aligned}
$$

In one more step, we notice the fact

$$
\begin{aligned}
\theta^{1-\alpha} & \leq\left(C+\frac{1-\alpha}{2} \int_{0}^{1} \theta^{-\frac{1+\alpha}{2}} \theta_{y} d y\right)^{2} \\
& \leq C+\frac{(1-\alpha)^{2}}{2} \int_{0}^{1} \frac{\alpha \theta_{y}^{2}}{v \theta^{1+\alpha}} d y \int_{0}^{1} \frac{v}{\alpha} d y \\
& \leq C+\frac{(1-\alpha)^{2} C}{2 \alpha} \int_{0}^{1} \frac{\alpha \theta_{y}^{2}}{v \theta^{1+\alpha}} d y .
\end{aligned}
$$

Then we get

$$
\int_{0}^{t} \int_{0}^{1} p u_{y} \theta^{-\alpha} d y d s \leq C+\frac{1}{2} \int_{0}^{t} \int_{0}^{1} \frac{u_{y}^{2}}{v \theta^{\alpha}} d y d s+\frac{(1-\alpha)^{2} C}{2 \alpha} \int_{0}^{1} \frac{\alpha \theta_{y}^{2}}{v \theta^{1+\alpha}} d y .
$$


Hence, if $\beta$ is suitably close to 1 , such that $\frac{(1-\alpha)^{2}}{2 \alpha} C<\frac{1}{2}, 0<\beta<\alpha<1$, we have

$$
\int_{0}^{t} \int_{0}^{1} \frac{\alpha \theta_{y}^{2}}{v \theta^{\alpha+1}} d y d s+\int_{0}^{t} \int_{0}^{1} \frac{u_{y}^{2}+\left|\mathbf{w}_{y}\right|^{2}+v(v)\left|\mathbf{b}_{y}\right|^{2}}{v \theta^{\alpha}} d y d s+\int_{0}^{t} \max _{y \in[0,1]} \theta^{1-\alpha} d s \leq C
$$

Combining Lemma 2.1 and Lemma 2.2, we can get the upper bound of the specific volume.

Lemma 2.3 There exists a positive constant $C>0$ such that, for any $t \in[0, T]$,

$$
\begin{aligned}
& \max _{y \in[0,1]} v(y, t) \leq C, \\
& \int_{0}^{t} \max _{y \in[0,1]} \theta^{2-\alpha} d s \leq C,
\end{aligned}
$$

where $0<\beta<\alpha<1$. Moreover, we have

$$
\int_{0}^{t} \int_{0}^{1}\left(u_{y}^{2}+\left|\mathbf{w}_{y}\right|^{2}+\left|\mathbf{b}_{y}\right|^{2}\right) d y d s \leq C
$$

Proof Let $0<\beta<\alpha<1$, where $\beta$ is given in Lemma 2.2. With the help of the Sobolev embedding theorem $W^{1,1}([0,1]) \hookrightarrow L^{\infty}([0,1])$, we get

$$
\begin{aligned}
\int_{0}^{t} \max _{y \in[0,1]}|\mathbf{b}|^{2} d s & \leq C \int_{0}^{t} \int_{0}^{1}|\mathbf{b}|^{2} d y d s+C \int_{0}^{t} \int_{0}^{1}\left|\mathbf{b} \cdot \mathbf{b}_{y}\right| d y d s \\
& \leq C+C \int_{0}^{t} \int_{0}^{1} \frac{v(v)\left|\mathbf{b}_{y}\right|^{2}}{v \theta} d y d s+\delta \int_{0}^{t} \int_{0}^{1} \frac{v \theta|\mathbf{b}|^{2}}{v(v)} d y d s \\
& \leq C+C \delta \int_{0}^{t} \max _{y \in[0,1]}|\mathbf{b}|^{2} d s,
\end{aligned}
$$

where in the second inequality, we have used (1.19) and (2.2). Then we have

$$
\int_{0}^{t} \max _{y \in[0,1]}|\mathbf{b}|^{2} d s \leq C
$$

We use the fact that

$$
\begin{aligned}
\theta^{\frac{1}{2}}(y, t) & \leq \int_{0}^{1} \theta^{\frac{1}{2}}(y, t) d y+\int_{0}^{1} \theta^{-\frac{1}{2}}(y, t)\left|\theta_{y}\right| d y \\
& \leq C+C\left(\int_{0}^{1} \frac{\theta_{y}^{2}}{v \theta^{2}} d y\right)^{\frac{1}{2}}\left(\int_{0}^{1} v \theta d y\right)^{\frac{1}{2}} \\
& \leq C+C\left(\max _{y \in[0,1]} v \int_{0}^{1} \frac{\theta_{y}^{2}}{v \theta^{2}} d y\right)^{\frac{1}{2}} .
\end{aligned}
$$

It is clear that

$$
\max _{y \in[0,1]} \theta \leq C+C \max _{y \in[0,1]} v \int_{0}^{1} \frac{\theta_{y}^{2}}{v \theta^{2}} d y .
$$


Substituting the above estimates into (2.12), we can get

$$
\max _{y \in[0,1]} v \leq C+C \int_{0}^{t} \theta d s \leq C+C \int_{0}^{t} \max _{y \in[0,1]} v \int_{0}^{1} \frac{\theta_{y}^{2}}{v \theta^{2}} d y d s
$$

By using Gronwall's inequality, we have

$$
\max _{y \in[0,1]} v \leq C+\exp \left(\int_{0}^{t} \int_{0}^{1} \frac{\theta_{y}^{2}}{v \theta^{2}} d y d s\right)
$$

which implies

$$
\max _{y \in[0,1]} v \leq C
$$

Next, we give the proof of (2.29). Since $\int_{0}^{1} \theta d y \leq C$, there exists $a_{2}(t)$ such that $\theta\left(a_{2}(t), t\right) \leq C$ for any $t \in[0, T]$. Therefore, with the help of (2.20) and (2.37), we have

$$
\begin{aligned}
\int_{0}^{t} \max _{y \in[0,1]} \theta^{2-\alpha} d s & =\int_{0}^{t}\left(\max _{y \in[0,1]} \int_{a_{2}(t)}^{y}\left|\theta^{-\frac{\alpha}{2}} \theta_{y}\right| d y+\theta^{\frac{2-\alpha}{2}}\left(a_{2}(s), s\right)\right)^{2} d s \\
& \leq C+\int_{0}^{t}\left(\int_{0}^{1}\left|\theta^{-\frac{\alpha}{2}} \theta_{y}\right| d y\right)^{2} d s \\
& \leq C+\int_{0}^{t}\left(\int_{0}^{1} \frac{\alpha \theta_{y}^{2}}{v \theta^{1+\alpha}} d y\right)\left(\int_{0}^{1} v \theta d y\right) d s \\
& \leq C .
\end{aligned}
$$

At last, we prove the estimate (2.30). Integrating the temperature equation (1.8) over $[0,1] \times[0, T]$, with the help of $(2.38)$, we get

$$
\begin{aligned}
\int_{0}^{t} \int_{0}^{1} \frac{u_{y}^{2}+\left|\mathbf{w}_{y}\right|^{2}+v(v)\left|\mathbf{b}_{y}\right|^{2}}{v} d y d s= & \left(\int_{0}^{1} \theta d y-\int_{0}^{1} \theta_{0} d y\right)+\int_{0}^{t} \int_{0}^{1} p u_{y} d y d s \\
\leq & C+\int_{0}^{t}\left(\int_{0}^{1} \frac{u_{y}^{2}}{v} d y\right)^{\frac{1}{2}}\left(\int_{0}^{1} p^{2} v d y\right)^{\frac{1}{2}} d s \\
\leq & C+\delta \int_{0}^{t} \int_{0}^{1} \frac{u_{y}^{2}}{v} d y d s \\
& +C(\delta) \int_{0}^{t} \max _{y \in[0,1]} \theta \int_{0}^{1} \theta d y d s \\
\leq & C+\delta \int_{0}^{t} \int_{0}^{1} \frac{u_{y}^{2}}{v} d y d s .
\end{aligned}
$$

From Lemma 2.1, we have

$$
\int_{0}^{t} \int_{0}^{1}\left(u_{y}^{2}+\left|\mathbf{w}_{y}\right|^{2}+\left|\mathbf{b}_{y}\right|^{2}\right) d y d s \leq C
$$




\section{A priori estimates in $H^{1}$ space}

In this section, we mainly derive the estimates on the $L^{2}$-norm of $v_{y}$ in Lemma 3.1. Then, by the standard energy method, estimates on the first-order derivatives of solutions are given in Lemmas 3.2-3.3 and the upper bound of $\theta$ is obtained in Remark 3.1.

Due to the restriction of the narrow range of $\alpha$ in the entropy estimate, we cannot obtain the bound of $\left\|v_{y}(t)\right\|_{L^{2}}$ directly, which is essential in deriving the upper bound of the temperature and the higher-order estimates of solutions. To overcome such difficulties, in the following lemma, we give a new representation of $\left\|v_{y}(t)\right\|_{L^{2}}$ for the planar MHD equations, which is motivated by Kanel"s work [22] for isentropic Navier-Stokes equations. In addition, we propose some new estimates to deal with the cross term of the fluid and the magnetic field.

Lemma 3.1 There exists a positive constant $C>0$ such that, for any $t \in[0, T]$,

$$
\begin{aligned}
& \int_{0}^{1}\left(\theta^{2}+u^{4}+|\mathbf{w}|^{4}+v|\mathbf{b}|^{4}\right) d y+\int_{0}^{t} \int_{0}^{1}\left(\theta_{y}^{2}+\theta u_{y}^{2}+\theta\left|\mathbf{w}_{y}\right|^{2}+\theta\left|\mathbf{b}_{y}\right|^{2}\right) d y d s \leq C, \\
& \int_{0}^{1} v_{y}^{2} d y+\int_{0}^{t} \int_{0}^{1} \theta v_{y}^{2} d y d s \leq C .
\end{aligned}
$$

Proof The proof will be divided into three steps.

Step 1. $L^{2}$ estimate of $v_{y}$.

First, we rewrite momentum equation $(1.6)_{2}$ as

$$
\left(\frac{v_{y}}{v}\right)_{t}=u_{t}+\left(p+\frac{1}{2}|\mathbf{b}|^{2}\right)_{y}
$$

Multiplying the above equation by $\frac{v_{y}}{v}$, we can get

$$
\frac{1}{2}\left(\left(\frac{v_{y}}{v}\right)^{2}\right)_{t}=\left(\frac{v_{y}}{v} u\right)_{t}-\left((\ln v)_{t} u\right)_{y}+\frac{u_{y}^{2}}{v}+\frac{v_{y} \theta_{y}}{v^{2}}-\frac{v_{y}^{2} \theta}{v^{3}}+\frac{v_{y} \mathbf{b} \cdot \mathbf{b}_{y}}{v} .
$$

Integrating the above result over $[0,1] \times[0, t]$, with the help of $(2.30)$, we obtain

$$
\begin{aligned}
\int_{0}^{1} & \left(\frac{1}{2}\left(\frac{v_{y}}{v}\right)^{2}-\frac{v_{y}}{v} u\right)(y, t) d y-\int_{0}^{1}\left(\frac{1}{2}\left(\frac{v_{y}}{v}\right)^{2}-\frac{v_{y}}{v} u\right)(y, 0) d y \\
= & \int_{0}^{t} \int_{0}^{1} \frac{u_{y}^{2}}{v} d y d s+\int_{0}^{t} \int_{0}^{1} \frac{v_{y} \theta_{y}}{v^{2}} d y d s \\
& -\int_{0}^{t} \int_{0}^{1} \frac{\theta v_{y}^{2}}{v^{3}} d y d s+\int_{0}^{t} \int_{0}^{1} \frac{v_{y} \mathbf{b} \cdot \mathbf{b}_{y}}{v} d y d s \\
\leq & C-\frac{1}{2} \int_{0}^{t} \int_{0}^{1} \frac{\theta v_{y}^{2}}{v^{3}} d y d s+\int_{0}^{t} \int_{0}^{1} \frac{\theta_{y}^{2}}{v \theta} d y d s \\
& +\int_{0}^{t} \int_{0}^{1}\left|\mathbf{b}_{y}\right|^{2} d y d s+\int_{0}^{t} \int_{0}^{1} \frac{|\mathbf{b}|^{2} v_{y}^{2}}{v^{2}} d y d s \\
\leq & C(\delta)-\frac{1}{2} \int_{0}^{t} \int_{0}^{1} \frac{\theta v_{y}^{2}}{v^{3}} d y d s+\delta \int_{0}^{t} \int_{0}^{1} \theta_{y}^{2} d y d s+\int_{0}^{t} \max _{y \in[0,1]}|\mathbf{b}|^{2} \int_{0}^{1} \frac{v_{y}^{2}}{v^{2}} d y .
\end{aligned}
$$


Then we have

$$
\begin{aligned}
& \int_{0}^{1} v_{y}^{2} d y+\int_{0}^{t} \int_{0}^{1} \frac{\theta v_{y}^{2}}{v^{3}} d y d s \\
& \quad \leq C(\delta)+\delta \int_{0}^{t} \int_{0}^{1} \theta_{y}^{2} d y d s+C \int_{0}^{t} \max _{y \in[0,1]}|\mathbf{b}|^{2} \int_{0}^{1} v_{y}^{2} d y .
\end{aligned}
$$

Step 2. Estimations of $\left\|\theta_{y}\right\|_{L^{2}\left(0, T ; L^{2}\right)}$.

In order to estimate the right-hand side of the above inequality, we multiply (1.8) by $\theta$, and integrate the resulting identity over $[0, t] \times \Omega$ to find

$$
\begin{aligned}
& \int_{0}^{t} \int_{0}^{1} \theta_{s} \theta d y d s+\int_{0}^{t} \int_{0}^{1} \frac{\theta_{y}^{2}}{v} d y d s \\
& \quad=-\int_{0}^{t} \int_{0}^{1} \frac{\theta^{2} u_{y}}{v} d y d s+\int_{0}^{t} \int_{0}^{1}\left(\frac{u_{y}^{2}}{v}+\frac{\left|\mathbf{w}_{y}\right|^{2}}{v}+\frac{v(v)\left|\mathbf{b}_{y}\right|^{2}}{v}\right) \theta d y d s .
\end{aligned}
$$

Furthermore, in order to estimate the last term on the right-hand side of the above equation, we multiply Eqs. $(1.6)_{2},(1.6)_{3},(1.6)_{4}$ by $2 u \theta, 2 \mathbf{w} \theta$ and $2 \mathbf{b} \theta$, respectively, and integrate the resulting identity over $[0, t] \times \Omega$, and we have

$$
\begin{aligned}
\int_{0}^{1}( & \left.\theta^{2}+u^{2} \theta+|\mathbf{w}|^{2} \theta+|\mathbf{b}|^{2} v \theta\right) d y \\
& +\int_{0}^{t} \int_{0}^{1}\left(\frac{\theta_{y}^{2}}{v}+\frac{\theta u_{y}^{2}}{v}+\frac{\theta\left|\mathbf{w}_{y}\right|^{2}}{v}\right) d y d s+\int_{0}^{t} \int_{0}^{1} \frac{v(v) \theta\left|\mathbf{b}_{y}\right|^{2}}{v} d y d s \\
\leq & C+\int_{0}^{t} \int_{0}^{1}\left(\frac{\theta^{2} u_{y}}{v}+\frac{2 \theta \theta_{y} u}{v}-\frac{2 u_{y} \theta_{y} u}{v}\right) d y d s \\
& -\int_{0}^{t} \int_{0}^{1}\left(2 \mathbf{b} \cdot \mathbf{b}_{y} u \theta d y d s+\int_{0}^{t} \int_{0}^{1} u^{2} \theta_{s}\right) d y d s \\
& +\int_{0}^{t} \int_{0}^{1}\left(2 \mathbf{b}_{y} \cdot \mathbf{w} \theta-\frac{2 \mathbf{w} \cdot \mathbf{w}_{y} \theta_{y}}{v}\right) d y d s+\int_{0}^{t} \int_{0}^{1}|\mathbf{w}|^{2} \theta_{s} d y d s \\
& +\int_{0}^{t} \int_{0}^{1}\left(-|\mathbf{b}|^{2} u_{y} \theta-\frac{2 v(v) \mathbf{b}_{y} \cdot \mathbf{b} \theta_{y}}{v}+2 \mathbf{w}_{y} \cdot \mathbf{b} \theta\right) d y d s+\int_{0}^{t} \int_{0}^{1}|\mathbf{b}|^{2} v \theta_{s} d y d s \\
\leq & C+H_{1}+H_{2}+H_{3}+H_{4}+H_{5}+H_{6}+H_{7} .
\end{aligned}
$$

Next, we will estimate the terms on the right-hand side of the above equation one by one. First, it follows from the bounds of the specific volume that, for any $\delta>0$,

$$
\begin{aligned}
H_{1} \leq & \delta \int_{0}^{t} \int_{0}^{1} \theta u_{y}^{2} d y d s+C(\delta) \int_{0}^{t} \int_{0}^{1} \theta^{3} d y d s \\
& +\delta \int_{0}^{t} \int_{0}^{1} \theta_{y}^{2} d y d s+C(\delta) \int_{0}^{t} \int_{0}^{1} u^{2} \theta^{2} d y d s+C(\delta) \int_{0}^{t} \int_{0}^{1} u^{2} u_{y}^{2} d y d s \\
\leq & \delta \int_{0}^{t} \int_{0}^{1} \theta u_{y}^{2} d y d s+\delta \int_{0}^{t} \int_{0}^{1} \theta_{y}^{2} d y d s \\
& +C(\delta) \int_{0}^{t} \max _{y \in \Omega} \theta^{2} d s+C(\delta) \int_{0}^{t} \int_{0}^{1} u^{2} u_{y}^{2} d y d s,
\end{aligned}
$$


Li and Shang Boundary Value Problems

(2019) 2019:162

Page 17 of 31

where in the last inequality we have used

$$
\begin{gathered}
\int_{0}^{t} \int_{0}^{1} u^{2} \theta^{2} d y d s \leq \int_{0}^{t} \max _{y \in \Omega} \theta^{2} \int_{0}^{1} u^{2} d y d s \leq C \int_{0}^{t} \max _{y \in \Omega} \theta^{2} d s, \\
H_{2} \leq \int_{0}^{t} \int_{0}^{1}|\mathbf{b}|^{2}\left|\mathbf{b}_{y}\right|^{2} d y d s+C \int_{0}^{t} \int_{0}^{1} u^{2} \theta^{2} d y d s \\
\quad \leq \int_{0}^{t} \int_{0}^{1}|\mathbf{b}|^{2}\left|\mathbf{b}_{y}\right|^{2} d y d s+C \int_{0}^{t} \max _{y \in \Omega} \theta^{2} d s,
\end{gathered}
$$

and

$$
\begin{aligned}
H_{3} \leq & C\left(\int_{0}^{t} \int_{0}^{1} u^{2} u_{y}^{2} d y d s+\int_{0}^{t} \int_{0}^{1} u^{2}\left|\mathbf{w}_{y}\right|^{2} d y d s+\int_{0}^{t} \int_{0}^{1} u^{2}\left|\mathbf{b}_{y}\right|^{2} d y d s\right. \\
& \left.+\int_{0}^{t} \int_{0}^{1} u^{2}\left(\frac{\theta_{y}}{v}\right)_{y} d y d s+\int_{0}^{t} \int_{0}^{1} u^{2} \theta^{2} d y d s\right) \\
\leq & \delta \int_{0}^{t} \int_{0}^{1} \theta_{y}^{2} d y d s+C(\delta) \int_{0}^{t} \int_{0}^{1} u^{2} u_{y}^{2} d y d s+C \int_{0}^{t} \int_{0}^{1} u^{2}\left|\mathbf{w}_{y}\right|^{2} d y d s \\
& +C \int_{0}^{t} \int_{0}^{1} u^{2}\left|\mathbf{b}_{y}\right|^{2} d y d s+C \int_{0}^{t} \max _{y \in \Omega} \theta^{2} d s .
\end{aligned}
$$

Next we address the term

$$
\begin{aligned}
H_{4} \leq & C \int_{0}^{t} \int_{0}^{1}\left|\mathbf{b}_{y}\right|^{2} d y d s+C \int_{0}^{t} \int_{0}^{1}|\mathbf{w}|^{2} \theta^{2} d y d s \\
& +\delta \int_{0}^{t} \int_{0}^{1} \theta_{y}^{2} d y d s+C(\delta) \int_{0}^{t} \int_{0}^{1}|\mathbf{w}|^{2}\left|\mathbf{w}_{y}\right|^{2} d y d s \\
\leq & C+\delta \int_{0}^{t} \int_{0}^{1} \theta_{y}^{2} d y d s+C(\delta) \int_{0}^{t} \int_{0}^{1}|\mathbf{w}|^{2}\left|\mathbf{w}_{y}\right|^{2} d y d s+C \int_{0}^{t} \max _{y \in \Omega} \theta^{2} d s .
\end{aligned}
$$

Similar to the estimation of $H_{3}$, we have

$$
\begin{aligned}
H_{5} \leq & \delta \int_{0}^{t} \int_{0}^{1} \theta_{y}^{2} d y d s+C \int_{0}^{t} \int_{0}^{1}|\mathbf{w}|^{2} u_{y}^{2} d y d s \\
& +C(\delta) \int_{0}^{t} \int_{0}^{1}|\mathbf{w}|^{2}\left|\mathbf{w}_{y}\right|^{2} d y d s+C \int_{0}^{t} \int_{0}^{1}|\mathbf{w}|^{2}\left|\mathbf{b}_{y}\right|^{2} d y d s \\
& +C \int_{0}^{t} \max _{y \in \Omega} \theta^{2} d s
\end{aligned}
$$

and

$$
\begin{aligned}
H_{6} \leq & C+\delta \int_{0}^{t} \int_{0}^{1} \theta_{y}^{2} d y d s+C \int_{0}^{t} \int_{0}^{1}|\mathbf{b}|^{2} u_{y}^{2} d y d s \\
& +C(\delta) \int_{0}^{t} \int_{0}^{1}|\mathbf{b}|^{2}\left|\mathbf{b}_{y}\right|^{2} d y d s+C \int_{0}^{t} \max _{y \in \Omega} \theta^{2} d s .
\end{aligned}
$$


The last term can be estimated as

$$
\begin{aligned}
H_{7} \leq & \delta \int_{0}^{t} \int_{0}^{1} \frac{\theta_{y}^{2}}{v} d y d s+C(\delta)\left(\int_{0}^{t} \int_{0}^{1}|\mathbf{b}|^{2} u_{y}^{2} d y d s+\int_{0}^{t} \int_{0}^{1}|\mathbf{b}|^{2}\left|\mathbf{w}_{y}\right|^{2} d y d s\right. \\
& \left.+\int_{0}^{t} \int_{0}^{1}|\mathbf{b}|^{2}\left|\mathbf{b}_{y}\right|^{2} d y d s+\int_{0}^{t}\|\mathbf{b}\|_{L^{\infty}}^{4} \int_{0}^{1} \frac{v_{y}^{2}}{v^{2}} d y d s+\int_{0}^{t} \max _{y \in \Omega} \theta^{2} d s\right) .
\end{aligned}
$$

We substitute (3.9)-(3.15) into (3.8) and choose $\delta$ suitably small to obtain

$$
\begin{aligned}
\int_{0}^{1}( & \left.\theta^{2}+u^{2} \theta+|\mathbf{w}|^{2} \theta+|\mathbf{b}|^{2} v \theta\right) d y+\int_{0}^{t} \int_{0}^{1} \theta_{y}^{2}+\theta u_{y}^{2}+\theta\left|\mathbf{w}_{y}\right|^{2}+\theta\left|\mathbf{b}_{y}\right|^{2} d y d s \\
\leq & C\left(1+\int_{0}^{t} \int_{0}^{1} u^{2} u_{y}^{2} d y d s+\int_{0}^{t} \int_{0}^{1}|\mathbf{b}|^{2}\left|\mathbf{b}_{y}\right|^{2} d y d s+\int_{0}^{t} \int_{0}^{1} u^{2}\left|\mathbf{w}_{y}\right|^{2} d y d s\right. \\
& +\int_{0}^{t} \int_{0}^{1} u^{2}\left|\mathbf{b}_{y}\right|^{2} d y d s+\int_{0}^{t} \int_{0}^{1}|\mathbf{w}|^{2}\left|\mathbf{b}_{y}\right|^{2} d y d s+\int_{0}^{t} \int_{0}^{1}|\mathbf{w}|^{2}\left|\mathbf{w}_{y}\right|^{2} d y d s \\
& +\int_{0}^{t} \int_{0}^{1}|\mathbf{w}|^{2} u_{y}^{2} d y d s+\int_{0}^{t} \int_{0}^{1}|\mathbf{b}|^{2} u_{y}^{2} d y d s+\int_{0}^{t} \int_{0}^{1}|\mathbf{b}|^{2}\left|\mathbf{w}_{y}\right|^{2} d y d s \\
& \left.+\int_{0}^{t}\|\mathbf{b}\|_{L^{\infty}}^{4} \int_{0}^{1} v_{y}^{2} d y d s+\int_{0}^{t} \max _{y \in \Omega} \theta^{2} d s\right) .
\end{aligned}
$$

Step 3. Estimate on the cross term of the fluid and the magnetic field.

In order to estimate the terms on the right-hand side of inequality (3.15), we multiply $(1.6)_{2}$ by $u^{3}$ and integrate the resulting equality over $\Omega \times(0, t)$ to get

$$
\begin{aligned}
& \frac{1}{4} \int_{0}^{1} u^{4} d y+3 \int_{0}^{t} \int_{0}^{1} \frac{u^{2} u_{y}^{2}}{v} d y d s \\
& \quad \leq C+\int_{0}^{t} \int_{0}^{1} \theta u^{2} u_{y} d y d s-\int_{0}^{t} \int_{0}^{1}\left(\frac{|\mathbf{b}|^{2}}{2}\right)_{y} u^{3} d y d s
\end{aligned}
$$

where

$$
\begin{aligned}
\int_{0}^{t} \int_{0}^{1} \theta u^{2} u_{y} d y d s & \leq \delta \int_{0}^{t} \int_{0}^{1} u^{2} u_{y}^{2} d y d s+C(\delta) \int_{0}^{t} \int_{0}^{1} u^{2} \theta^{2} d y d s \\
& \leq \delta \int_{0}^{t} \int_{0}^{1} u^{2} u_{y}^{2} d y d s+C(\delta) \int_{0}^{t} \max _{y \in \Omega} \theta^{2} d s
\end{aligned}
$$

and

$$
\begin{aligned}
\int_{0}^{t} \int_{0}^{1}\left(\frac{|\mathbf{b}|^{2}}{2}\right)_{y} u^{3} d y d s & \leq \delta \int_{0}^{t} \int_{0}^{1} u^{2} u_{y}^{2} d y d s+C(\delta) \int_{0}^{t} \int_{0}^{1} u^{2}|\mathbf{b}|^{4} d y d s \\
& \leq \delta \int_{0}^{t} \int_{0}^{1} u^{2} u_{y}^{2} d y d s+C(\delta) \int_{0}^{t}\|\mathbf{b}\|_{L^{\infty}}^{4} d s \\
& \leq C(\delta)+\delta \int_{0}^{t} \int_{0}^{1} u^{2} u_{y}^{2} d y d s .
\end{aligned}
$$

Then we have

$$
\int_{0}^{1} u^{4} d y+\int_{0}^{t} \int_{0}^{1} u^{2} u_{y}^{2} d y d s \leq C+C \int_{0}^{t} \max _{y \in \Omega} \theta^{2} d s
$$


Similarly, multiply $(1.6)_{3}$ by $|\mathbf{w}|^{2} \mathbf{w}$, and integrate the resulting identity over $[0, t] \times \Omega$, and we get

$$
\begin{aligned}
& \frac{1}{4} \int_{0}^{1}|\mathbf{w}|^{4} d y+\int_{0}^{t} \int_{0}^{1} \frac{|\mathbf{w}|^{2}\left|\mathbf{w}_{y}\right|^{2}}{v} d y d s \\
& \quad \leq C+\int_{0}^{t} \int_{0}^{1} \mathbf{b}_{y} \cdot|\mathbf{w}|^{2} \mathbf{w} d y d s \\
& \quad \leq C+\delta \int_{0}^{t} \int_{0}^{1}|\mathbf{w}|^{2}\left|\mathbf{b}_{y}\right|^{2} d y d s+C(\delta) \int_{0}^{t} \int_{0}^{1}|\mathbf{w}|^{4} d y d s \\
& \quad \leq C(\delta)+\delta \int_{0}^{t} \int_{0}^{1}|\mathbf{w}|^{2}\left|\mathbf{b}_{y}\right|^{2} d y d s .
\end{aligned}
$$

Multiply (1.6) $)_{4}$ by $|\mathbf{b}|^{2} \mathbf{b}$ and integrate the resulting identity over $[0, t] \times \Omega$; we obtain

$$
\begin{aligned}
\frac{1}{4} \int_{0}^{1} v|\mathbf{b}|^{4} d y+\int_{0}^{t} \int_{0}^{1} \frac{v(v)|\mathbf{b}|^{2}|\mathbf{b} y|^{2}}{v} d y d s \\
\leq C-\frac{3}{4} \int_{0}^{t} \int_{0}^{1} u_{y}|\mathbf{b}|^{4} d y d s+C \int_{0}^{t} \int_{0}^{1} \mathbf{w}_{y} \cdot|\mathbf{b}|^{2} \mathbf{b} d y d s \\
\leq C+\delta \int_{0}^{t} \int_{0}^{1}|\mathbf{b}|^{2} u_{y}^{2} d y d s+C(\delta) \int_{0}^{t} \int_{0}^{1}|\mathbf{b}|^{6} d y d s+\delta \int_{0}^{t} \int_{0}^{1}|\mathbf{b}|^{2}\left|\mathbf{w}_{y}\right|^{2} d y d s \\
\quad+C(\delta) \int_{0}^{t} \int_{0}^{1}|\mathbf{b}|^{4} d y d s \\
\leq C(\delta)+\delta \int_{0}^{t} \int_{0}^{1}|\mathbf{b}|^{2} u_{y}^{2} d y d s+\delta \int_{0}^{t} \int_{0}^{1}|\mathbf{b}|^{2}\left|\mathbf{w}_{y}\right|^{2} d y d s .
\end{aligned}
$$

Furthermore, multiplying (1.6) $)_{2}$ by $u|\mathbf{w}|^{2}$ and (1.6) $)_{3}$ by $u^{2} \mathbf{w}$, integrating the resulting identity over $[0, t] \times \Omega$, adding the results equation together we have

$$
\begin{aligned}
\frac{1}{2} \int_{0}^{1} u^{2}|\mathbf{w}|^{2} d y+\int_{0}^{t} \int_{0}^{1} \frac{|\mathbf{w}|^{2} u_{y}^{2}}{v} d y d s+\int_{0}^{t} \int_{0}^{1} \frac{u^{2}\left|\mathbf{w}_{y}\right|^{2}}{v} d y d s \\
\leq C+\int_{0}^{t} \int_{0}^{1}\left|\mathbf{b}_{y} \cdot u^{2} \mathbf{w}\right| d y d s+C \int_{0}^{t} \int_{0}^{1}\left|u u_{y} \mathbf{w} \cdot \mathbf{w}_{y}\right| d y d s \\
\quad-\int_{0}^{t} \int_{0}^{1}\left(p+\frac{1}{2}|\mathbf{b}|^{2}\right)_{y} u|\mathbf{w}|^{2} d y d s \\
\leq C\left(1+\int_{0}^{t} \int_{0}^{1}\left|\mathbf{b}_{y}\right|^{2} d y d s+\int_{0}^{t}\|u\|_{L^{\infty}}^{4} d s+\int_{0}^{t} \int_{0}^{1} u^{2} u_{y}^{2} d y d s\right. \\
\left.\quad+\int_{0}^{t} \int_{0}^{1}|\mathbf{w}|^{2}\left|\mathbf{w}_{y}\right|^{2} d y d s-\int_{0}^{t} \int_{0}^{1}\left(p+\frac{1}{2}|\mathbf{b}|^{2}\right)_{y} u|\mathbf{w}|^{2} d y d s\right),
\end{aligned}
$$

where the last term on the right-hand side of the above equation can be estimated as

$$
\begin{aligned}
& \int_{0}^{t} \int_{0}^{1}\left(p+\frac{1}{2}|\mathbf{b}|^{2}\right)_{y} u|\mathbf{w}|^{2} d y d s \\
& \quad=-\int_{0}^{t} \int_{0}^{1} \frac{\theta}{v}\left(u_{y}|\mathbf{w}|^{2}+2 u \mathbf{w} \cdot \mathbf{w}_{y}\right) d y d s+\int_{0}^{t} \int_{0}^{1} \mathbf{b} \cdot \mathbf{b}_{y} u|\mathbf{w}|^{2} d y d s
\end{aligned}
$$


Li and Shang Boundary Value Problems

(2019) 2019:162

Page 20 of 31

$$
\begin{aligned}
\leq & \delta \int_{0}^{t} \int_{0}^{1}|\mathbf{w}|^{2} u_{y}^{2} d y d s+\delta \int_{0}^{t} \int_{0}^{1}|\mathbf{w}|^{2}\left|\mathbf{w}_{y}\right|^{2} d y d s \\
& +\delta \int_{0}^{t} \int_{0}^{1} u^{2}\left|\mathbf{b}_{y}\right|^{2} d y d s+C(\delta) \int_{0}^{t} \int_{0}^{1}|\mathbf{b}|^{2}|\mathbf{w}|^{4} d y d s+C(\delta) \int_{0}^{t} \max _{y \in \Omega} \theta^{2} d s \\
\leq & C(\delta)+\delta \int_{0}^{t} \int_{0}^{1}|\mathbf{w}|^{2} u_{y}^{2} d y d s+\delta \int_{0}^{t} \int_{0}^{1}|\mathbf{w}|^{2}\left|\mathbf{w}_{y}\right|^{2} d y d s \\
& +\delta \int_{0}^{t} \int_{0}^{1} u^{2}\left|\mathbf{b}_{y}\right|^{2} d y d s+C(\delta) \int_{0}^{t} \max _{y \in \Omega} \theta^{2} d s,
\end{aligned}
$$

then, combining (3.20) and (3.21), we have

$$
\begin{aligned}
& \int_{0}^{1} u^{2}|\mathbf{w}|^{2} d y+\int_{0}^{t} \int_{0}^{1}|\mathbf{w}|^{2} u_{y}^{2} d y d s+\int_{0}^{t} \int_{0}^{1} u^{2}\left|\mathbf{w}_{y}\right|^{2} d y d s \\
& \quad \leq C+\delta \int_{0}^{t} \int_{0}^{1}|\mathbf{w}|^{2}\left|\mathbf{b}_{y}\right|^{2} d y d s+\delta \int_{0}^{t} \int_{0}^{1} u^{2}\left|\mathbf{b}_{y}\right|^{2} d y d s+C(\delta) \int_{0}^{t} \max _{y \in \Omega} \theta^{2} d s .
\end{aligned}
$$

Multiplying (1.6) 2 by $u v|\mathbf{b}|^{2}$ and (1.6) $)_{4}$ by $u^{2} \mathbf{b}$, integrating the resulting identity over $[0, t] \times \Omega$ and adding the resulting equation together, we have

$$
\begin{aligned}
& \frac{1}{2} \int_{0}^{1} v u^{2}|\mathbf{b}|^{2} d y+\int_{0}^{t} \int_{0}^{1} \frac{v(v) u^{2}\left|\mathbf{b}_{y}\right|^{2}}{v} d y d s+\int_{0}^{t} \int_{0}^{1}|\mathbf{b}|^{2} u_{y}^{2} d y d s \\
& \leq \delta \int_{0}^{t} \int_{0}^{1} u^{2}\left|\mathbf{w}_{y}\right|^{2} d y d s+\delta \int_{0}^{t} \int_{0}^{1}|\mathbf{b}|^{2} u_{y}^{2} d y d s \\
& \quad+C(\delta)\left(1+\int_{0}^{t} \int_{0}^{1} u^{2} u_{y}^{2} d y d s+\int_{0}^{t} \int_{0}^{1}|\mathbf{b}|^{2}\left|\mathbf{b}_{y}\right|^{2} d y d s+\int_{0}^{t} \int_{0}^{1}|\mathbf{b}|^{4} v_{y}^{2} d y d s\right. \\
& \left.\quad-\int_{0}^{t} \int_{0}^{1}\left(p+\frac{1}{2}|\mathbf{b}|^{2}\right)_{y} v u|\mathbf{b}|^{2} d y d s\right),
\end{aligned}
$$

where the last term on the right-hand side of the above equation can be estimated as

$$
\begin{aligned}
& \int_{0}^{t} \int_{0}^{1}\left(p+\frac{1}{2}|\mathbf{b}|^{2}\right)_{y} v u|\mathbf{b}|^{2} d y d s \\
& =\int_{0}^{t} \int_{0}^{1}\left(\theta_{y} u|\mathbf{b}|^{2}-\frac{v_{y} \theta u|\mathbf{b}|^{2}}{v}\right) d y d s+\int_{0}^{t} \int_{0}^{1} u v|\mathbf{b}|^{2} \mathbf{b} \cdot \mathbf{b}_{y} d y d s \\
& \leq \delta \int_{0}^{t} \int_{0}^{1} \theta_{y}^{2} d y d s+C(\delta) \int_{0}^{t} \int_{0}^{1} u^{2}|\mathbf{b}|^{4} d y d s+C \int_{0}^{t} \int_{0}^{1} \theta^{2} u^{2} d y d s \\
& \quad+\int_{0}^{t} \int_{0}^{1}|\mathbf{b}|^{2}\left|\mathbf{b}_{y}\right|^{2} d y d s+C \int_{0}^{t} \int_{0}^{1}|\mathbf{b}|^{4} v_{y}^{2} d y d s \\
& \leq C(\delta)+\delta \int_{0}^{t} \int_{0}^{1} \theta_{y}^{2} d y d s+\delta \int_{0}^{t} \int_{0}^{1}|\mathbf{b}|^{2}\left|\mathbf{b}_{y}\right|^{2} d y d s+C \int_{0}^{t}\|\mathbf{b}\|_{L^{\infty}}^{4} \int_{0}^{1} v_{y}^{2} d y d s \\
& \quad+C \int_{0}^{t} \max _{y \in \Omega} \theta^{2} d s .
\end{aligned}
$$


Then, combining (3.26) and (3.27), we have

$$
\begin{aligned}
& \frac{1}{2} \int_{0}^{1} v u^{2}|\mathbf{b}|^{2} d y+\int_{0}^{t} \int_{0}^{1} \frac{u^{2}\left|\mathbf{b}_{y}\right|^{2}}{v} d y d s+\int_{0}^{t} \int_{0}^{1}|\mathbf{b}|^{2} u_{y}^{2} d y d s \\
& \leq \delta \int_{0}^{t} \int_{0}^{1} u^{2}\left|\mathbf{w}_{y}\right|^{2} d y d s+\delta \int_{0}^{t} \int_{0}^{1}|\mathbf{b}|^{2}\left|\mathbf{w}_{y}\right|^{2} d y d s+\delta \int_{0}^{t} \int_{0}^{1} \theta_{y}^{2} d y d s \\
& \quad+C(\delta)\left(1+\int_{0}^{t}\|\mathbf{b}\|_{L^{\infty}}^{4} \int_{0}^{1} v_{y}^{2} d y d s+\int_{0}^{t} \max _{y \in \Omega} \theta^{2} d s\right) .
\end{aligned}
$$

Similarly to (3.26), multiplying (1.6) 3 by $v \mathbf{w}|\mathbf{b}|^{2}$ and (1.6) 4 by $|\mathbf{w}|^{2} \mathbf{b}$, integrating the resulting identity over $[0, t] \times \Omega$ and adding them together, we have

$$
\begin{aligned}
& \frac{1}{2} \int_{0}^{1} v|\mathbf{w}|^{2}|\mathbf{b}|^{2} d y+\int_{0}^{t} \int_{0}^{1} \frac{v(v)|\mathbf{w}|^{2}\left|\mathbf{b}_{y}\right|^{2}}{v} d y d s+\int_{0}^{t} \int_{0}^{1}|\mathbf{b}|^{2}\left|\mathbf{w}_{y}\right|^{2} d y d s \\
& \leq C+\left.\int_{0}^{t} \int_{0}^{1}\left|\mathbf{w}_{y} \cdot\right| \mathbf{w}\right|^{2} \mathbf{b}\left|d y d s+\int_{0}^{t} \int_{0}^{1}\right| u_{y}|\mathbf{w}|^{2}|\mathbf{b}|^{2} \mid d y d s \\
&+\int_{0}^{t} \int_{0}^{1}\left|\mathbf{w} \cdot \mathbf{w}_{y} \mathbf{b} \cdot \mathbf{b}_{y}\right| d y d s+\left.\int_{0}^{t} \int_{0}^{1}\left|\mathbf{w} \cdot \mathbf{w}_{y} v_{y}\right| \mathbf{b}\right|^{2} \mid d y d s \\
&+\left.\int_{0}^{t} \int_{0}^{1}|\mathbf{b} \cdot v \mathbf{w}| \mathbf{b}\right|^{2} \mid d y d s \\
& \leq \delta \int_{0}^{t} \int_{0}^{1}|\mathbf{b}|^{2}\left|\mathbf{w}_{y}\right|^{2} d y d s+\delta \int_{0}^{t} \int_{0}^{1}|\mathbf{b}|^{2} u_{y}^{2} d y d s+\delta \int_{0}^{t} \int_{0}^{1}|\mathbf{w}|^{2}\left|\mathbf{b}_{y}\right|^{2} d y d s \\
&+C(\delta)\left(1+\int_{0}^{t} \int_{0}^{1}|\mathbf{w}|^{2}\left|\mathbf{w}_{y}\right|^{2} d y d s+\int_{0}^{t} \int_{0}^{1}|\mathbf{b}|^{2}\left|\mathbf{b}_{y}\right|^{2} d y d s+\int_{0}^{t} \int_{0}^{1}|\mathbf{b}|^{4} v_{y}^{2} d y d s\right) \\
& \leq 2 \delta \int_{0}^{t} \int_{0}^{1}|\mathbf{b}|^{2}\left|\mathbf{w}_{y}\right|^{2} d y d s+2 \delta \int_{0}^{t} \int_{0}^{1}|\mathbf{b}|^{2} u_{y}^{2} d y d s+2 \delta \int_{0}^{t} \int_{0}^{1}|\mathbf{w}|^{2}\left|\mathbf{b}_{y}\right|^{2} d y d s \\
&+C(\delta)\left(1+\int_{0}^{t}\|\mathbf{b}\|_{L^{\infty}}^{4} \int_{0}^{1} v_{y}^{2} d y d s\right),
\end{aligned}
$$

where in the last inequality we have used (2.2), (2.40), (3.21), (3.22) and the bounds of $v$.

From the above estimates (3.8)-(3.29), we finally have

$$
\begin{aligned}
\int_{0}^{1}( & \left.\theta^{2}+u^{4}+|\mathbf{w}|^{4}+|\mathbf{b}|^{4}+|\mathbf{b}|^{4}\right) d y+\int_{0}^{1}\left(u^{2}|\mathbf{w}|^{2}+u^{2}|\mathbf{b}|^{2}+|\mathbf{w}|^{2}|\mathbf{b}|^{2}\right) d y \\
& +\int_{0}^{t} \int_{0}^{1}\left(\theta_{y}^{2}+u_{y}^{2}+\left|\mathbf{w}_{y}\right|^{2}+\left|\mathbf{b}_{y}\right|^{2}\right) d y d s \\
& +\int_{0}^{t} \int_{0}^{1}\left(u^{2} u_{y}^{2}+|\mathbf{w}|^{2}\left|\mathbf{w}_{y}\right|^{2}+|\mathbf{b}|^{2}\left|\mathbf{b}_{y}\right|^{2}\right) d y d s \\
& +\int_{0}^{t} \int_{0}^{1}\left(u^{2}\left|\mathbf{w}_{y}\right|^{2}+|\mathbf{w}|^{2} u_{y}^{2}+u^{2}\left|\mathbf{b}_{y}\right|^{2}+|\mathbf{w}|^{2}\left|\mathbf{b}_{y}\right|^{2}\right) d y d s \\
& +\int_{0}^{t} \int_{0}^{1}\left(|\mathbf{b}|^{2} u_{y}^{2}+|\mathbf{b}|^{2}\left|\mathbf{w}_{y}\right|^{2}+|\mathbf{b}|^{2}\left|\mathbf{b}_{y}\right|^{2} d y d s+|\mathbf{b}|^{2}\left|\mathbf{b}_{y}\right|^{2}\right) d y d s \\
\leq & C\left(1+\int_{0}^{t}\|\mathbf{b}\|_{L^{\infty}}^{4} \int_{0}^{1} v_{y}^{2} d y d s+\int_{0}^{t} \max _{y \in \Omega} \theta^{2} d s\right) .
\end{aligned}
$$


Li and Shang Boundary Value Problems

(2019) 2019:162

Page 22 of 31

By standard calculations, the last term on the right-hand side of (3.30) yields, for any $\delta>0$,

$$
\begin{aligned}
\int_{0}^{t} \max _{y \in \Omega} \theta^{2} d s & \leq C+\int_{0}^{t} \max _{y \in \Omega}\left(\int_{a_{2}(s)}^{y} \theta_{y} d y\right)^{2} d s \\
& \leq C+\int_{0}^{t}\left(\int_{\Omega} \frac{\theta_{y}^{2}}{\theta} d y \int_{\Omega} \theta d y\right) d s \\
& \leq C+\delta \int_{0}^{t} \int_{0}^{1} \theta_{y}^{2} d y d s+C(\delta) \int_{0}^{t} \int_{0}^{1} \frac{\theta_{y}^{2}}{\theta^{2}} d y d s \\
& \leq C(\delta)+\delta \int_{0}^{t} \int_{0}^{1} \theta_{y}^{2} d y d s .
\end{aligned}
$$

Then we have

$$
\int_{0}^{1} \theta^{2} d y+\int_{0}^{t} \int_{0}^{1} \theta_{y}^{2} d y d s \leq C+C \int_{0}^{t}\|\mathbf{b}\|_{L^{\infty}}^{4} \int_{0}^{1} v_{y}^{2} d y d s .
$$

Combining with (3.6) we obtain

$$
\int_{0}^{1} v_{y}^{2} d y+\int_{0}^{t} \int_{0}^{1} \theta v_{y}^{2} d y d s \leq C+C \int_{0}^{t}\left\|\mathbf{b}_{y}\right\|_{L^{2}}^{2} \int_{0}^{1} v_{y}^{2} d y
$$

by using Gronwall's inequality, this proves Lemma 3.1

Next, we give the estimations of the first-order derivatives of $u, \mathbf{w}, \mathbf{b}$ and $\theta$ in Lemmas 3.2-3.3.

Lemma 3.2 There exists a positive constant $C>0$ such that, for any $t \in[0, T]$,

$$
\int_{0}^{1}\left(\left|\mathbf{w}_{y}\right|^{2}+\left|\mathbf{b}_{y}\right|^{2}\right) d y+\int_{0}^{T} \int_{0}^{1}\left(\left|\mathbf{w}_{y y}\right|^{2}+\left|\mathbf{b}_{y y}\right|^{2}+\left|\mathbf{w}_{t}\right|^{2}+\left|\mathbf{b}_{t}\right|^{2}\right) d y d t \leq C .
$$

Proof Multiplying $(1.6)_{3}$ with $-\mathbf{w}_{y y}$, integrating the result over $[0,1] \times[0, t]$, we have

$$
\begin{aligned}
& \frac{1}{2} \int_{0}^{1}\left|\mathbf{w}_{y}(y, t)\right|^{2} d y-\frac{1}{2} \int_{0}^{1}\left|\mathbf{w}_{y}(y, 0)\right|^{2} d y \\
& \quad=-\int_{0}^{t} \int_{0}^{1} \frac{\left|\mathbf{w}_{y y}\right|^{2}}{v} d y d s+\int_{0}^{t} \int_{0}^{1} \frac{v_{y} \mathbf{w}_{y} \cdot \mathbf{w}_{y y}}{v^{2}} d y d s-\int_{0}^{t} \int_{0}^{1} \mathbf{b}_{y} \cdot \mathbf{w}_{y y} d y d s \\
& \quad \leq-\int_{0}^{t} \int_{0}^{1} \frac{\left|\mathbf{w}_{y y}\right|^{2}}{v} d y d s+C \int_{0}^{t} \int_{0}^{1}\left|\left(\mathbf{b}_{y}+v_{y} \mathbf{w}_{y}\right) \mathbf{w}_{y y}\right| d y d s \\
& \quad \leq-\frac{3}{4} \int_{0}^{t} \int_{0}^{1} \frac{\left|\mathbf{w}_{y y}\right|^{2}}{v} d y d s+C \int_{0}^{t} \int_{0}^{1}\left|\mathbf{b}_{y}\right|^{2} d y d s+C \int_{0}^{t} \max _{y \in[0,1]}\left|\mathbf{w}_{y}\right|^{2} \int_{0}^{1} v_{y}^{2} d y d s \\
& \quad \leq C-\frac{1}{2} \int_{0}^{t} \int_{0}^{1} \frac{\left|\mathbf{w}_{y y}\right|^{2}}{v} d y d s .
\end{aligned}
$$

It is easy to see that

$$
\int_{0}^{1}\left|\mathbf{w}_{y}\right|^{2} d y+\int_{0}^{t} \int_{0}^{1}\left|\mathbf{w}_{y y}\right|^{2} d y d s \leq C .
$$


Rewrite $(1.6)_{4}$ as

$$
\mathbf{b}_{t}=-\frac{u_{y}}{v} \mathbf{b}+\frac{1}{v}\left(\mathbf{w}+\frac{v(v) \mathbf{b}_{y}}{v}\right)_{y}
$$

Similar to the estimate on $\mathbf{w}$, multiplying the above equation by $-\mathbf{b}_{y y}$, integrating the result over $[0,1] \times[0, t]$, we have

$$
\begin{aligned}
\frac{1}{2} \int_{0}^{1}\left|\mathbf{b}_{y}(y, t)\right|^{2} d y-\frac{1}{2} \int_{0}^{1}\left|\mathbf{b}_{y}(y, 0)\right|^{2} d y \\
=\int_{0}^{t} \int_{0}^{1} \frac{u_{y} \mathbf{b}-\mathbf{w}_{y}}{v} \cdot \mathbf{b}_{y y} d y d s-\int_{0}^{t} \int_{0}^{1} \frac{v(v)\left|\mathbf{b}_{y y}\right|^{2}}{v^{2}} d y d s+\int_{0}^{t} \int_{0}^{1} \frac{v_{y} \mathbf{b}_{y} \cdot \mathbf{b}_{y y}}{v^{3}} d y d s \\
\quad-\int_{0}^{t} \int_{0}^{1} \frac{v^{\prime}(v) v_{y} \mathbf{b}_{y} \cdot \mathbf{b}_{y y}}{v^{2}} d y d s \\
\leq C-\frac{3}{4} \int_{0}^{t} \int_{0}^{1} \frac{v(v)\left|\mathbf{b}_{y y}\right|^{2}}{v^{2}} d y d s+C \int_{0}^{t} \int_{0}^{1}|\mathbf{b}|^{2} u_{y}^{2} d y d s+C \int_{0}^{t} \int_{0}^{1} v_{y}^{2}\left|\mathbf{b}_{y}\right|^{2} d y d s \\
\leq C-\frac{3}{4} \int_{0}^{t} \int_{0}^{1} \frac{v(v)\left|\mathbf{b}_{y y}\right|^{2}}{v^{2}} d y d s+C \max _{[0,1] \times[0, t]}|\mathbf{b}|^{2} \int_{0}^{t} \int_{0}^{1} u_{y}^{2} d y d s \\
\quad+C \int_{0}^{t} \max _{y \in[0,1]}\left|\mathbf{b}_{y}\right|^{2} \int_{0}^{1} v_{y}^{2} d y d s \\
\leq C-\frac{1}{2} \int_{0}^{t} \int_{0}^{1} \frac{v(v)\left|\mathbf{b}_{y y}\right|^{2}}{v^{2}} d y d s .
\end{aligned}
$$

It is clear that

$$
\int_{0}^{1}\left|\mathbf{b}_{y}\right|^{2} d y+\int_{0}^{t} \int_{0}^{1}\left|\mathbf{b}_{y y}\right|^{2} d y d s \leq C
$$

Next, we give the estimates of $\mathbf{w}_{t}$ and $\mathbf{b}_{t}$ from (1.6) 3 and (3.37), respectively:

$$
\begin{aligned}
\int_{0}^{t} \int_{0}^{1}\left|\mathbf{w}_{s}\right|^{2} d y d s & \leq 2 \int_{0}^{t} \int_{0}^{1}\left|\mathbf{b}_{y}\right|^{2} d y d s+2 \int_{0}^{t} \int_{0}^{1}\left(\frac{\mathbf{w}_{y y}}{v}-\frac{v_{y} \mathbf{w}_{y}}{v^{2}}\right)^{2} d y d s \\
& \leq C+C \int_{0}^{t} \int_{0}^{1}\left|\mathbf{w}_{y y}\right|^{2} d y d s+C \int_{0}^{t} \int_{0}^{1} v_{y}^{2}\left|\mathbf{w}_{y}\right|^{2} d y d s \\
& \leq C+C \int_{0}^{t} \max _{y \in[0,1]}\left|\mathbf{w}_{y}\right|^{2} \int_{0}^{1} v_{y}^{2} d y d s \leq C, \\
\int_{0}^{t} \int_{0}^{1} \mathbf{b}_{s}^{2} d y d s \leq & 2 \int_{0}^{t} \int_{0}^{1} \frac{\mathbf{b}^{2} u_{y}^{2}}{v} d y d s+2 \int_{0}^{t} \int_{0}^{1} \frac{1}{v^{2}}\left(\mathbf{w}+\frac{v(v) \mathbf{b}_{y}}{v}\right)_{y}^{2} d y d s \leq C .
\end{aligned}
$$

Lemma 3.3 There exists a positive constant $C>0$ such that, for any $t \in[0, T]$,

$$
\int_{0}^{1}\left(u_{y}^{2}+\theta_{y}^{2}\right) d y+\int_{0}^{T} \int_{0}^{1}\left(u_{y y}^{2}+\theta_{y y}^{2}+u_{t}^{2}+\theta_{t}^{2}\right) d y d t \leq C .
$$


Li and Shang Boundary Value Problems

(2019) 2019:162

Page 24 of 31

Proof Multiplying $(1.6)_{2}$ by $-u_{y y}$, integrating the results over $[0,1] \times[0, t]$, we have

$$
\begin{aligned}
\frac{1}{2} & \int_{0}^{1} u_{y}^{2}(y, t) d y-\frac{1}{2} \int_{0}^{1} u_{y}^{2}(y, 0) d y \\
& =\int_{0}^{t} \int_{0}^{1}\left(p+\frac{1}{2}|\mathbf{b}|^{2}\right)_{y} u_{y y} d y d s-\int_{0}^{t} \int_{0}^{1}\left(\frac{u_{y}}{v}\right)_{y} u_{y y} d y d s \\
& =\int_{0}^{t} \int_{0}^{1}\left(p+\frac{1}{2}|\mathbf{b}|^{2}\right)_{y} u_{y y} d y d s-\int_{0}^{t} \int_{0}^{1} \frac{u_{y y}^{2}}{v} d y d s+\int_{0}^{t} \int_{0}^{1} \frac{v_{y} u_{y} u_{y y}}{v^{2}} d y d s \\
& \leq C \int_{0}^{t} \int_{0}^{1}\left(p+\frac{1}{2}|\mathbf{b}|^{2}\right)_{y}^{2} d y d s-\frac{1}{2} \int_{0}^{t} \int_{0}^{1} \frac{u_{y y}^{2}}{v} d y d s+C \int_{0}^{t} \int_{0}^{1} v_{y}^{2} u_{y}^{2} d y d s,
\end{aligned}
$$

where

$$
\begin{aligned}
& \int_{0}^{t} \int_{0}^{1}\left(p+\frac{1}{2}|\mathbf{b}|^{2}\right)_{y}^{2} d y d s \\
& \quad=\int_{0}^{t} \int_{0}^{1}\left(\frac{\theta_{y}}{v}-\frac{v_{y} \theta}{v^{2}}+\mathbf{b} \cdot \mathbf{b}_{y}\right)^{2} d y d s \\
& \quad \leq C \int_{0}^{t} \int_{0}^{1} \theta_{y}^{2} d y d s+C \int_{0}^{t} \int_{0}^{1} \theta^{2} v_{y}^{2} d y d s+C \int_{0}^{t} \int_{0}^{1} \mathbf{b}^{2} \mathbf{b}_{y}^{2} d y d s \\
& \quad \leq C+C \int_{0}^{t} \max _{y \in[0,1]} \theta^{2} \int_{0}^{1} v_{y}^{2} d y d s \\
& \quad \leq C
\end{aligned}
$$

and

$$
\begin{aligned}
\int_{0}^{t} \int_{0}^{1} v_{y}^{2} u_{y}^{2} d y d s & \leq \int_{0}^{t} \max _{y \in[0,1]} u_{y}^{2} \int_{0}^{1} v_{y}^{2} d y d s \\
& \leq C(\delta) \int_{0}^{t} \int_{0}^{1} u_{y}^{2} d y d s+\delta \int_{0}^{t} \int_{0}^{1} u_{y y}^{2} d y d s \\
& \leq C(\delta)+\delta \int_{0}^{t} \int_{0}^{1} u_{y y}^{2} d y d s .
\end{aligned}
$$

Choosing sufficiently small $\delta$, we can get

$$
\int_{0}^{1} u_{y}^{2} d y+\int_{0}^{t} \int_{0}^{1} u_{y y}^{2} d y d s \leq C
$$

Furthermore, based on the momentum equation $(1.6)_{2}$,

$$
\int_{0}^{t} \int_{0}^{1} u_{s}^{2} d y d s \leq C \int_{0}^{t} \int_{0}^{1}\left(p+\frac{1}{2}|\mathbf{b}|^{2}\right)_{y}^{2} d y d s+C \int_{0}^{t} \int_{0}^{1}\left(\frac{u_{y}}{v}\right)_{y}^{2} d y d s \leq C .
$$


For the temperature equation, we multiply (1.8) by $-\theta_{y y}$ and integrate the results over $[0,1] \times[0, t]$, we arrive at

$$
\begin{aligned}
& \frac{1}{2} \int_{0}^{1} \theta_{y}^{2}(y, t) d y-\frac{1}{2} \int_{0}^{1} \theta_{y}^{2}(y, 0) d y \\
& \quad=\int_{0}^{t} \int_{0}^{1}\left(\frac{\theta u_{y} \theta_{y y}}{v}-\left(\frac{\theta_{y}}{v}\right)_{y} \theta_{y y}\right) d y d s-\int_{0}^{t} \int_{0}^{1}\left(\frac{u_{y}^{2}+\left|\mathbf{w}_{y}\right|^{2}+v(v)\left|\mathbf{b}_{y}\right|^{2}}{v}\right) \theta_{y y} d y d s \\
& \quad \leq-\frac{1}{2} \int_{0}^{t} \int_{0}^{1} \frac{\theta_{y y}^{2}}{v} d y d s+C \int_{0}^{t} \int_{0}^{1}\left(\theta^{2} u_{y}^{2}+\theta_{y}^{2} v_{y}^{2}+u_{y}^{4}+\left|\mathbf{w}_{y}\right|^{4}+\left|\mathbf{b}_{y}\right|^{4}\right) d y d s \\
& \quad \leq-\frac{1}{2} \int_{0}^{t} \int_{0}^{1} \frac{\theta_{y y}^{2}}{v} d y d s+C\left(\int_{0}^{t} \max _{y \in[0,1]} \theta^{2} \int_{0}^{1} u_{y}^{2} d y d s+\int_{0}^{t} \max _{y \in[0,1]} \theta_{y}^{2} \int_{0}^{1} v_{y}^{2} d y d s\right) \\
& \quad \leq C-\frac{1}{4} \int_{0}^{t} \int_{0}^{1} \frac{\theta_{y y}^{2}}{v} d y d s,
\end{aligned}
$$

from which we can get the estimate of $\theta_{t}$. Thus, we complete the proof of this lemma.

Remark 3.1 With the help of (3.42) and the Sobolev embedding theorem $W^{1,1}([0,1]) \hookrightarrow$ $L^{\infty}([0,1])$, we can obtain the positive upper bound of the temperature $\theta$. That is,

$$
\theta(y, t) \leq C \int_{0}^{1}|\theta| d y+C \int_{0}^{1}\left|\theta_{y}\right| d y \leq C\left(\int_{0}^{1} \theta^{2} d y\right)^{\frac{1}{2}}+C\left(\int_{0}^{1} \theta_{y}^{2} d y\right)^{\frac{1}{2}} \leq C
$$

\section{A priori estimates on the higher-order derivatives of solutions}

Finally, in this section, we derive the estimates on the higher-order derivatives of $u, \mathbf{w}, \mathbf{b}$ and $\theta$ one by one in Lemma 4.1 .

Lemma 4.1 There exists a positive constant $C>0$ such that, for any $t \in[0, T]$,

$$
\begin{aligned}
& \int_{0}^{1}\left(u_{t}^{2}+\left|\mathbf{w}_{t}\right|^{2}+u_{y y}^{2}+\left|\mathbf{w}_{y y}\right|^{2}\right) d y+\int_{0}^{T} \int_{0}^{1}\left(u_{y t}^{2}+\left|\mathbf{w}_{y t}\right|^{2}\right) d y d t \leq C, \\
& \int_{0}^{1}\left(\left|\mathbf{b}_{t}\right|^{2}+\theta_{t}^{2}+\left|\mathbf{b}_{y y}\right|^{2}+\theta_{y y}^{2}\right) d y+\int_{0}^{T} \int_{0}^{1}\left(\left|\mathbf{b}_{y t}\right|^{2}+\theta_{y t}^{2}\right) d y d t \leq C .
\end{aligned}
$$

Proof The proof will be split into three steps, which are concerned with the treatments of the velocity, magnetic field and temperature, respectively.

Step 1. Estimations of the velocity.

First, differentiating the momentum equation (1.6) 2 with respect to $t$, we get

$$
u_{t t}+\left(p+\frac{1}{2}|\mathbf{b}|^{2}\right)_{y t}=\left(\frac{u_{y}}{v}\right)_{y t}
$$

Multiplying the above equation with $u_{t}$, integrating the resulting equation over $[0,1] \times$ $[0, t]$ and using (3.34) and (3.42), we have

$$
\begin{aligned}
& \frac{1}{2} \int_{0}^{1} u_{t}^{2}(y, t) d y-\frac{1}{2} \int_{0}^{1} u_{t}^{2}(y, 0) d y \\
& \quad=\int_{0}^{t} \int_{0}^{1}\left(\frac{\theta_{s}}{v}-\frac{\theta u_{y}}{v^{2}}+\mathbf{b} \cdot \mathbf{b}_{s}-\frac{u_{y s}}{v}+\frac{u_{y}^{2}}{v^{2}}\right) u_{s y} d y d s
\end{aligned}
$$




$$
\begin{aligned}
& \leq-\frac{1}{2} \int_{0}^{t} \int_{0}^{1} \frac{u_{y s}^{2}}{v} d y d s+C \int_{0}^{t} \int_{0}^{1}\left(u_{y}^{4}+\theta^{2} u_{y}^{2}+\theta_{s}^{2}+|\mathbf{b}|^{2}\left|\mathbf{b}_{s}\right|^{2}\right) d y d s \\
& \leq-\frac{1}{2} \int_{0}^{t} \int_{0}^{1} \frac{u_{y s}^{2}}{v} d y d s+C .
\end{aligned}
$$

Thus, we obtain

$$
\int_{0}^{1} u_{t}^{2} d y+\int_{0}^{t} \int_{0}^{1} \frac{u_{y s}^{2}}{v} d y d s \leq C .
$$

On the other hand, rewrite $(1.6)_{2}$ as

$$
\frac{u_{y y}}{v}=u_{t}+\left(p+\frac{1}{2}|\mathbf{b}|^{2}\right)_{y}+\frac{u_{y} v_{y}}{v^{2}} .
$$

Using (3.49) and (4.5), we have

$$
\begin{aligned}
\int_{0}^{1} \frac{u_{y y}^{2}}{v^{2}} d y & \leq C \int_{0}^{1}\left(u_{t}^{2}+\theta_{y}^{2}+v_{y}^{2} \theta^{2}+|\mathbf{b}|^{2}\left|\mathbf{b}_{y}\right|^{2}+v_{y}^{2} u_{y}^{2}\right) d y \\
& \leq C \max _{y \in[0,1]} u_{y}^{2} \int_{0}^{1} v_{y}^{2} d y \\
& \leq C+\frac{1}{2} \int_{0}^{1} \frac{u_{y y}^{2}}{v^{2}} d y
\end{aligned}
$$

which implies $\int_{0}^{1} u_{y y}^{2} d y \leq C$, for any $t \in[0, T]$.

Differentiating equation $(1.6)_{3}$ with respect to $t$, multiplying the result by $\mathbf{w}_{t}$, integrating it over $[0,1] \times[0, t]$ and using (3.34), we have

$$
\begin{aligned}
& \frac{1}{2} \int_{0}^{1}\left|\mathbf{w}_{t}\right|^{2}(y, t) d y-\frac{1}{2} \int_{0}^{1}\left|\mathbf{w}_{t}\right|^{2}(y, 0) d y \\
& \quad=-\int_{0}^{t} \int_{0}^{1} \mathbf{b}_{s} \mathbf{w}_{s y} d y d s-\int_{0}^{t} \int_{0}^{1} \frac{\left|\mathbf{w}_{y s}\right|^{2}}{v} d y d s+\int_{0}^{t} \int_{0}^{1} \frac{\mathbf{w}_{y} u_{y} \mathbf{w}_{y s}}{v^{2}} d y d s \\
& \quad \leq-\frac{1}{2} \int_{0}^{t} \int_{0}^{1} \frac{\left|\mathbf{w}_{y s}\right|^{2}}{v} d y d s+C \int_{0}^{t} \int_{0}^{1} \mathbf{b}_{s}^{2} d y d s+C \int_{0}^{t} \int_{0}^{1} \mathbf{w}_{y}^{2} u_{y}^{2} d y d s \\
& \quad \leq C-\frac{1}{2} \int_{0}^{t} \int_{0}^{1} \frac{\mathbf{w}_{y s}^{2}}{v} d y d s,
\end{aligned}
$$

which gives

$$
\int_{0}^{1}\left|\mathbf{w}_{s}\right|^{2} d y+\int_{0}^{t} \int_{0}^{1}\left|\mathbf{w}_{y s}\right|^{2} d y d s \leq C .
$$

\section{Step 2. Estimations of the magnetic field.}

Now, we turn to the estimate of $\mathbf{b}$. Similarly, differentiating equation $(1.6)_{4}$ with respect to $t$, multiplying the result by $(v \mathbf{b})_{t}$, integrating over $[0,1] \times[0, t]$ and using $(4.9)$, we arrive 
at

$$
\begin{aligned}
\frac{1}{2} \int_{0}^{1}(v \mathbf{b})_{t}^{2}(y, t) d y-\frac{1}{2} \int_{0}^{1}(v \mathbf{b})_{t}^{2}(y, 0) d y \\
\quad=\int_{0}^{t} \int_{0}^{1} \mathbf{w}_{y s} \cdot(v \mathbf{b})_{s} d y d s-\int_{0}^{t} \int_{0}^{1}\left(\frac{v(v) \mathbf{b}_{y}}{v}\right)_{s} \cdot(v \mathbf{b})_{s y} d y d s \\
\leq \int_{0}^{t} \int_{0}^{1}\left|\mathbf{w}_{y s}\right|^{2} d y d s+C \int_{0}^{t} \int_{0}^{1} v^{2}\left|\mathbf{b}_{s}\right|^{2} d y d s+C \int_{0}^{t} \int_{0}^{1}|\mathbf{b}|^{2} u_{y}^{2} d y d s \\
\quad-\frac{1}{2} \int_{0}^{t} \int_{0}^{1} v(v)\left|\mathbf{b}_{y s}\right|^{2} d y d s+C \int_{0}^{t} \int_{0}^{1}\left(|\mathbf{b}|^{2} u_{y y}^{2}+u_{y}^{2}\left|\mathbf{b}_{y}\right|^{2}+v_{y}^{2}\left|\mathbf{b}_{s}\right|^{2}\right) d y d s \\
\leq C-\frac{1}{2} \int_{0}^{t} \int_{0}^{1} v(v)\left|\mathbf{b}_{y s}\right|^{2} d y d s+C \int_{0}^{t} \max _{y \in[0,1]}\left|\mathbf{b}_{s}\right|^{2} \int_{0}^{1} v_{y}^{2} d y d s \\
\leq C-\frac{1}{4} \int_{0}^{t} \int_{0}^{1} v(v)\left|\mathbf{b}_{y s}\right|^{2} d y d s .
\end{aligned}
$$

Considering the fact that $\mathbf{b}_{t}=\frac{(v \mathbf{b})_{t}}{v}-\frac{v_{t} \mathbf{b}}{v}$, we can get

$$
\int_{0}^{1}\left|\mathbf{b}_{t}\right|^{2} d y+\int_{0}^{t} \int_{0}^{1}\left|\mathbf{b}_{y s}\right|^{2} d y d s \leq C
$$

Based on (1.6) 3 , using (3.34) and (4.9) we have

$$
\begin{aligned}
\int_{0}^{1}\left(\frac{\mathbf{w}_{y y}}{v}\right)^{2} d y & \leq C \int_{0}^{1}\left(\left|\mathbf{w}_{t}\right|^{2}+\left|\mathbf{b}_{y}\right|^{2}+\frac{\left|\mathbf{w}_{y}\right|^{2} v_{y}^{2}}{v^{4}}\right) d y \\
& \leq C+C \max _{y \in[0,1]}\left|\mathbf{w}_{y}\right|^{2} \int_{0}^{1} v_{y}^{2} d y \\
& \leq C+\frac{1}{2} \int_{0}^{1}\left(\frac{\mathbf{w}_{y y}}{v}\right)^{2} d y
\end{aligned}
$$

which gives $\int_{0}^{1}\left|\mathbf{w}_{y y}\right|^{2} d y \leq C$.

Then from (1.6) 4 , using (3.34) and (4.11) we have

$$
\begin{aligned}
\int_{0}^{1}\left(\frac{v(v) \mathbf{b}_{y y}}{v}\right)^{2} d y & \leq C \int_{0}^{1}\left(v^{2}\left|\mathbf{b}_{t}\right|^{2}+u_{y}^{2}|\mathbf{b}|^{2}+\left|\mathbf{w}_{y}\right|^{2}+\left|\mathbf{b}_{y}\right|^{2} v_{y}^{2}\right) d y \\
& \leq C+C \max _{y \in[0,1]}\left|\mathbf{b}_{y}\right|^{2} \int_{0}^{1} v_{y}^{2} d y \\
& \leq C+\frac{1}{2} \int_{0}^{1}\left(\frac{v(v) \mathbf{b}_{y y}}{v}\right)^{2} d y
\end{aligned}
$$

which implies $\int_{0}^{1}\left|\mathbf{b}_{y y}\right|^{2} d y \leq C$.

Step 3. Estimations of the temperature. 
At last, differentiating the temperature equation (1.8) with respect to $t$, multiplying the result by $\theta_{t}$, integrating over $[0,1] \times[0, t]$ and using Lemma 3.2, Lemma 3.3, we have

$$
\begin{aligned}
\frac{1}{2} \int_{0}^{1} \theta_{t}^{2}(y, t) d y-\frac{1}{2} \int_{0}^{1} \theta_{t}^{2}(y, 0) d y \\
=-\int_{0}^{t} \int_{0}^{1}\left(\frac{\theta u_{y}}{v}\right)_{s} \theta_{s} d y d s-\int_{0}^{t} \int_{0}^{1}\left(\frac{\theta_{y}}{v}\right)_{s} \theta_{y s} d y d s \\
\quad+\int_{0}^{t} \int_{0}^{1}\left(\frac{u_{y}^{2}+\mathbf{w}_{y}^{2}+v(v) \mathbf{b}_{y}^{2}}{v}\right)_{s} \theta_{s} d y d s \\
\leq C-\int_{0}^{t} \int_{0}^{1}\left(\frac{\theta_{y s}}{v}-\frac{\theta_{y} u_{y}}{v^{2}}\right) \theta_{y s} d y d s \\
\leq C-\frac{1}{2} \int_{0}^{t} \int_{0}^{1} \frac{\theta_{y s}^{2}}{v} d y d s,
\end{aligned}
$$

which gives

$$
\int_{0}^{1} \theta_{t}^{2} d y+\int_{0}^{t} \int_{0}^{1} \theta_{y t}^{2} d y d s \leq C
$$

Furthermore, rewrite the temperature equation (1.8) as

$$
\frac{\theta_{y y}}{v}=\theta_{t}+\frac{\theta}{v} u_{y}+\frac{v_{y} \theta_{y}}{v^{2}}-\frac{u_{y}^{2}+\left|\mathbf{w}_{y}\right|^{2}+v(v)\left|\mathbf{b}_{y}\right|^{2}}{v} .
$$

Integrating the above equation respect to $y$ over $[0,1]$ and using (4.15), we have

$$
\begin{aligned}
\int_{0}^{1} \theta_{y y}^{2} d y & \leq C \int_{0}^{1}\left(\theta_{t}^{2}+\theta^{2} u_{y}^{2}+v_{y}^{2} \theta_{y}^{2}+v_{y}^{2}+u_{y}^{4}+\left|\mathbf{w}_{y}\right|^{4}+\left|\mathbf{b}_{y}\right|^{4}\right) d y \\
& \leq C+C \max _{y \in[0,1]} \theta_{y}^{2} \int_{0}^{1} v_{y}^{2} d y \\
& \leq C+\frac{1}{2} \int_{0}^{1} \theta_{y y}^{2} d y,
\end{aligned}
$$

which gives $\int_{0}^{1} \theta_{y y}^{2} d y \leq C$. This completes the proof of this lemma.

Thus, Theorem 1.1 can be proved by the method of continuation, i.e., extending the local-in-time solution to the global one through the a priori global estimates. If the initial data are Hölder continuous, we prove the global existence for classical solutions; we refer to [6] for details.

\section{Global strong solution for planar Hall-MHD equations}

In this section, we prove that, for the planar Hall-MHD equations (1.28) with initialboundary conditions (1.9) and (1.10), similar results in Theorem 1.1 can be obtained by using the techniques of this paper with some modifications, which is claimed in Remark 1.5. First, we give a brief derivation of model (1.28) and start from the following 
three-dimensional Hall-MHD equations (see [36] for details):

$$
\left\{\begin{array}{l}
\rho_{t}+\operatorname{div}(\rho \mathbf{u})=0, \quad x \in \mathbb{R}^{3}, t>0, \\
(\rho \mathbf{u})_{t}+\operatorname{div}(\rho \mathbf{u} \otimes \mathbf{u})+\nabla p=(\nabla \times \mathbf{B}) \times \mathbf{B}+\operatorname{div} \mathbb{T}, \\
\mathbf{B}_{t}-\nabla \times(\mathbf{u} \times \mathbf{B})=-\nabla \times(\nu \nabla \times \mathbf{B})-\nabla \times\left(\frac{\epsilon(\nabla \times \mathbf{B}) \times \mathbf{B}}{\rho}\right), \quad \operatorname{div} \mathbf{B}=0, \\
\mathcal{E}_{t}+\operatorname{div}\left(\mathbf{u}\left(\mathcal{E}^{\prime}+p\right)\right)=\operatorname{div}((\mathbf{u} \times \mathbf{B}) \times \mathbf{B}+\nu \mathbf{B} \times(\nabla \times \mathbf{B})) \\
\quad+\operatorname{div}\left(\mathbf{B} \times\left(\frac{\epsilon(\nabla \times \mathbf{B}) \times \mathbf{B}}{\rho}\right)+u \mathbb{T}+\kappa \nabla \theta\right),
\end{array}\right.
$$

where $\epsilon$ is a constant denoting Hall parameter. When one deals with phenomena with characteristic length scales comparable or smaller than the ion skin depth, the Hall effect, which takes into account the relation between electrons and ions, cannot be neglected.

Then, by the same method as planar MHD equations in the introduction, we have the following planar Hall-MHD equations in Euler coordinates:

$$
\left\{\begin{array}{l}
\rho_{t}+(\rho u)_{x}=0, \quad x \in \Omega, t>0, \\
(\rho u)_{t}+\left(\rho u^{2}+p+\frac{1}{2}|\mathbf{b}|^{2}\right)_{x}=\left(\lambda u_{x}\right)_{x}, \\
(\rho \mathbf{w})_{t}+(\rho u \mathbf{w}-\mathbf{b})_{x}=\left(\mu \mathbf{w}_{x}\right)_{x}, \\
\mathbf{b}_{t}+(u \mathbf{b}-\mathbf{w})_{x}=\left(\nu \mathbf{b}_{x}\right)_{x}+\left(\frac{\epsilon A \mathbf{b}_{x}}{\rho}\right)_{x}, \\
\mathcal{E}_{t}+\left(u\left(\mathcal{E}+p+\frac{1}{2}|\mathbf{b}|^{2}\right)-\mathbf{w} \cdot \mathbf{b}\right)_{x} \\
\quad=\left(\lambda u u_{x}+\mu \mathbf{w} \cdot \mathbf{w}_{x}+\nu \mathbf{b} \cdot \mathbf{b}_{x}+\frac{\epsilon A^{\top} \mathbf{b} \cdot \mathbf{b}_{x}}{\rho}+\kappa \theta_{x}\right)_{x},
\end{array}\right.
$$

from which we deduce (1.28) after a Lagrangian transformation. The key observation in system (1.28) is that, after direct calculation, the temperature equation (1.8) also holds for the planar Hall-MHD equations. Hence, (1.28) reduces to

$$
\left\{\begin{array}{l}
v_{t}+u_{y}=0 \\
u_{t}+\left(p+\frac{1}{2}|\mathbf{b}|^{2}\right)_{y}=\left(\frac{\lambda u_{y}}{v}\right)_{y}, \\
\mathbf{w}_{t}-\mathbf{b}_{y}=\left(\frac{\mu \mathbf{w}_{y}}{v}\right)_{y}, \\
(v \mathbf{b})_{t}-\mathbf{w}_{y}=\left(\frac{v \mathbf{b}_{y}}{v}\right)_{y}+\left(\epsilon A \mathbf{b}_{y}\right)_{y}, \\
\theta_{t}+p u_{y}=\frac{\lambda}{v} u_{y}^{2}+\frac{\mu}{v}\left|\mathbf{w}_{y}\right|^{2}+\frac{v}{v}\left|\mathbf{b}_{y}\right|^{2}+\left(\frac{\kappa \theta_{y}}{v}\right)_{y}
\end{array}\right.
$$

where the Hall term only appears in magnetic equation $(5.3)_{3}$.

Next, we show the global existence of strong solution for the planar Hall-MHD equations (5.3) with initial-boundary conditions (1.9) and (1.10). In fact, it follows from (1.28) $)_{1}$ and $(1.28)_{5}$ that one can get the conservation of mass (2.1) and energy (2.2), respectively. Then, applying the same method as in Lemma 2.1-2.3 to system (5.3), lower and upper bounds of the density can be obtained due to the magnetic equation $(5.3)_{3}$ but they will not be used in the proof. For the a priori estimates on the first-order and higher-order of the global strong solution, we refer the reader to Sect. 3 and Sect. 4 and only need to modify the places where the magnetic equation $(5.3)_{3}$ is involved. Hence, the global existence of a strong solution for the planar Hall-MHD equations (1.28) with initial-boundary conditions (1.9) and (1.10) is proved. 


\section{Acknowledgements}

The authors would like to thank BVOP for considering this paper to be published and express their sincere thanks to the anonymous referee for careful reading and corrections of the paper.

\section{Funding}

The authors' research was supported in part by Chinese National Natural Science Foundation under grant 11571232 and 11831011.

\section{Availability of data and materials}

Not applicable.

\section{Competing interests}

The authors declare that they have no competing interests.

\section{Authors' contributions}

The authors read and approved the final manuscript.

\section{Publisher's Note}

Springer Nature remains neutral with regard to jurisdictional claims in published maps and institutional affiliations.

Received: 24 May 2019 Accepted: 3 October 2019 Published online: 21 October 2019

\section{References}

1. Abidi, H., Hmidi, T:. Résultats d'existence dans des espaces critiques pour le système de la MHD inhomogène. Ann. Math. Blaise Pascal 14(1), 103-148 (2007)

2. Abidi, H., Paicu, M.: Global existence for the magnetohydrodynamic system in critical spaces. Proc. R. Soc. Edinb., Sect. A 138(3), 447-476 (2008)

3. Blank, A.A.: Non-Linear Wave Propagation with Applications to Physics and Magnetohydrodynamics (A. Jeffrey and T. Taniuti). Academic press, San Diego (1964)

4. Brio, M., Wu, C.C.: An upwind differencing scheme for the equations of ideal magnetohydrodynamics. J. Comput. Phys. 75(2), 400-422 (1988)

5. Cabannes, H., Holt, M., Taussig, R.: Theoretical Magnetofluiddynamics. Academic Press, San Diego (1970)

6. Chen, G.-Q., Wang, D.: Global solutions of nonlinear magnetohydrodynamics with large initial data. J. Differ. Equ. 182(2), 344-376 (2002)

7. Chen, G.-Q., Wang, D.: Existence and continuous dependence of large solutions for the magnetohydrodynamic equations. Z. Angew. Math. Phys. 54(4), 608-632 (2003)

8. Clemmow, P.C., Dougherty, J.P., Barach, J.P.: Electrodynamics of Particles and Plasmas. Addison-Wesley, Reading (1990)

9. Dafermos, C.M.: Global smooth solutions to the initial-boundary value problem for the equations of one-dimensional nonlinear thermoviscoelasticity. SIAM J. Math. Anal. 13(3), 397-408 (1982)

10. Dafermos, C.M., Hsiao, L.: Global smooth thermomechanical processes in one-dimensional nonlinear thermoviscoelasticity. Nonlinear Anal. 6(5), 435-454 (1982)

11. Davidson, P.A.: Introduction to Magnetohydrodynamics, 2nd edn. Cambridge Texts in Applied Mathematics. Cambridge University Press, Cambridge (2017)

12. Duan, R., Jiang, F., Jiang, S.: On the Rayleigh-Taylor instability for incompressible, inviscid magnetohydrodynamic flows. SIAM J. Appl. Math. 71(6), 1990-2013 (2011)

13. Fan, J., Hu, Y.: Global strong solutions to the 1-D compressible magnetohydrodynamic equations with zero resistivity. J. Math. Phys. 56(2), 023101 (2015)

14. Fan, J., Huang, S., Li, F.: Global strong solutions to the planar compressible magnetohydrodynamic equations with large initial data and vacuum. Kinet. Relat. Models 10(4), 1035-1053 (2017)

15. Fan, J., Jiang, S., Nakamura, G.: Vanishing shear viscosity limit in the magnetohydrodynamic equations. Commun. Math. Phys. 270(3), 691-708 (2007)

16. Fan, J., Jiang, S., Nakamura, G.: Stability of weak solutions to equations of magnetohydrodynamics with Lebesgue initial data. J. Differ. Equ. 251(8), 2025-2036 (2011)

17. Freistühler, H., Szmolyan, P.: Existence and bifurcation of viscous profiles for all intermediate magnetohydrodynamic shock waves. SIAM J. Math. Anal. 26(1), 112-128 (1995)

18. Guo, B., Xie, B.: Global existence of weak solutions for generalized quantum MHD equation. Ann. Appl. Math. 33(2), $111-129(2017)$

19. Hoff, D., Tsyganov, E.: Uniqueness and continuous dependence of weak solutions in compressible magnetohydrodynamics. Z. Angew. Math. Phys. 56(5), 791-804 (2005)

20. Hu, Y., Ju, Q.: Global large solutions of magnetohydrodynamics with temperature-dependent heat conductivity. Z. Angew. Math. Phys. 66(3), 865-889 (2015)

21. Jiang, S.: On the asymptotic behavior of the motion of a viscous, heat-conducting, one-dimensional real gas. Math. $Z$. 216(2), 317-336 (1994)

22. Kanel', J.I.: A model system of equations for the one-dimensional motion of a gas. Differ. Uravn. 4, 721-734 (1968)

23. Kawashima, S., Okada, M.: Smooth global solutions for the one-dimensional equations in magnetohydrodynamics. Proc. Jpn. Acad., Ser. A, Math. Sci. 58(9), 384-387 (1982)

24. Kawashima, S., Shizuta, Y.: Magnetohydrodynamic approximation of the complete equations for an electromagnetic fluid. Tsukuba J. Math. 10(1), 131-149 (1986)

25. Kawohl, B.: Global existence of large solutions to initial-boundary value problems for a viscous, heat-conducting, one-dimensional real gas. J. Differ. Equ. 58(1), 76-103 (1985) 
26. Kazhikhov, A.V.: A priori estimates for the solutions of equations of magnetic-gas-dynamics, boundary value problems for equations of mathematical physics, Krasnoyarsk (1987) (in Russian)

27. Kazhikhov, A.V., Shelukhin, V.V.: Unique global solution with respect to time of initial-boundary value problems for one-dimensional equations of a viscous gas. Prikl. Mat. Meh. 41(2), 282-291 (1977)

28. Li, Y.: Global strong solutions to the one-dimensional heat-conductive model for planar non-resistive magnetohydrodynamics with large data. Z. Angew. Math. Phys. 69(3). Art. 78 (2018)

29. Li, Y.: Global well-posedness to the one-dimensional model for planar non-resistive magnetohydrodynamics with large data and vacuum. J. Math. Anal. Appl. 462(2), 1342-1356 (2018)

30. Li, Y., Jiang, L.: Global weak solutions for the Cauchy problem to one-dimensional heat-conductive mhd equations of viscous non-resistive gas. Acta Appl. Math. 163, 185-206 (2019)

31. Nash, J.: Le problème de Cauchy pour les équations différentielles d'un fluide général. Bull. Soc. Math. Fr. 90, 487-497 (1962)

32. Pan, R.: Global smooth solutions and the asymptotic behavior of the motion of a viscous, heat-conductive, one-dimensional real gas. J. Partial Differ. Equ. 11(3), 273-288 (1998)

33. Pan, R., Zhang, W.: Compressible Navier-Stokes equations with temperature dependent heat conductivity. Commun Math. Sci. 13(2), 401-425 (2015)

34. Polovin, R.V.R.V., Haar, D.T.: Fundamentals of Magnetohydrodynamics. Consultants Bureau, New York (1990)

35. Qin, X., Yang, T., Yao, Z.-A., Zhgou, W.: A study on the boundary layer for the planar magnetohydrodynamics system. Acta Math. Sci. Ser. B Engl. Ed. 35(4), 787-806 (2015)

36. Shang, Z:: Global existence and large time behavior of solutions for full compressible Hall-MHD equations. Appl. Anal. (2018). https://doi.org/10.1080/00036811.2018.1549320

37. $\mathrm{Si}, \mathrm{X}$., Ye, X.: Global well-posedness for the incompressible MHD equations with density-dependent viscosity and resistivity coefficients. Z. Angew. Math. Phys. 67(5), Art. 126 (2016)

38. Tani, A.: On the first initial-boundary value problem of the generalized Burgers' equation. Publ. Res. Inst. Math. Sci. 10(1), 209-233 (1974/75)

39. Vol'pert, A.I., Hudjaev, S.I.: The Cauchy problem for composite systems of nonlinear differential equations. Mat. Sb. (N.S.) 87(129), 504-528 (1972)

40. Wang, D.: Large solutions to the initial-boundary value problem for planar magnetohydrodynamics. SIAM J. Appl. Math. 63(4), 1424-1441 (2003)

41. Woods, L.C.: Principles of Magnetoplasma Dynamics. Oxford Science Publications. Clarendon, New York (1987)

42. Zhang, J., Xie, F.: Global solution for a one-dimensional model problem in thermally radiative magnetohydrodynamics. J. Differ. Equ. 245(7), 1853-1882 (2008)

43. Zhang, J., Zhao, X.: On the global solvability and the non-resistive limit of the one-dimensional compressible heat-conductive MHD equations. J. Math. Phys. 58(3), 031504 (2017)

\section{Submit your manuscript to a SpringerOpen ${ }^{\circ}$ journal and benefit from:}

- Convenient online submission

- Rigorous peer review

- Open access: articles freely available online

- High visibility within the field

- Retaining the copyright to your article

Submit your next manuscript at $>$ springeropen.com 